\title{
Transitional turbulent flow in a stenosed coronary artery with a physiological pulsatile flow
}

\author{
N. Freidoonimehr, M. Arjomandi, N. Sedaghatizadeh, R. Chin, and A. Zander \\ School of Mechanical Engineering \\ University of Adelaide, Adelaide, South Australia 5005, Australia
}

\begin{abstract}
The turbulence in the blood flow, caused by plaque deposition on the arterial wall, increases by the combined effect of the complex plaque geometries and the pulsatile blood flow. The correlation between the plaque geometry, the pulsatile inlet flow and the induced turbulence in a constricted artery is investigated in this study. Pressure drop, flow velocity and wall shear stress are determined for stenosed coronary artery models with three different degrees of asymmetric stenosis and for different heart working conditions. A Computational Fluid Dynamics model, validated against experimental data published in the literature, was developed to simulate the blood pulsatile flow inside a stenosed coronary artery model. The transitional flow behaviour was quantified by investigation of the changes in the turbulence kinetic energy. It was shown that the separation starts from the side of the asymmetric stenosis and spreads to its opposite side further downstream. The results suggest that there is a high risk of the formation of a secondary stenosis at a downstream distance equal to 10- times of the artery diameter at the side and bottom regions of the first stenosis due to the existence of the recirculation zones and low shear stresses. Finally, a stenosed patient specific coronary artery model was employed to illustrate the applicability of the obtained results for real geometry models. The results of this study provide a good prediction of pressure drop and blood flow rate, which can be applied in the investigation of the heart muscle workout and the required heart power.
\end{abstract}

Keywords: Pulsatile flow; artery plaque; transition; coronary artery; stenosis; turbulence

\section{Nomenclature}

A Cross sectional area $\left(\mathrm{m}^{2}\right)$

$D \quad$ Diameter $(m)$

$f \quad$ Flow variable

$N$ number of cycles

$P$ Pressure $(\mathrm{Pa})$

Re Reynolds number $\left(=\rho u_{c} D / \mu\right)$

$t \quad$ Time $(s)$

$T$ Cycle time period $(s)$

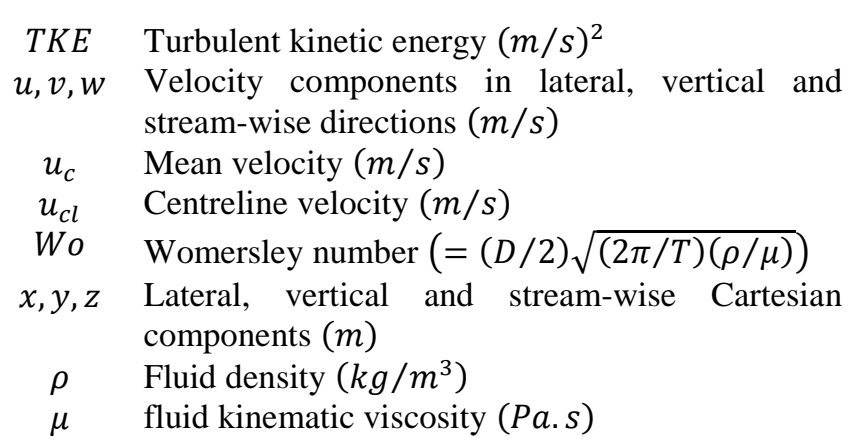

\section{Introduction}

Cardiovascular diseases (CVDs), a group of disorders related to the heart and blood vessels, are one of the main causes of death, contributing to more than half of the population deaths in

This is the author manuscript accepted for publication and has undergone full peer review but has not been through the copyediting, typesetting, pagination and proofreading process, which may lead to differences between this version and the Version of Record. Please cite this article as doi: $10.1002 / \mathrm{cnm} .3347$ 
2015. One of blood vessel disorders is atherosclerosis which is caused by plaque deposition on the arterial wall and may lead to the obstruction of the blood flow known as stenosis. Lipid, calcium, low-density lipoprotein (LDL), and a specific type of white blood cells (macrophage cell) are different components that can cause the formation of the plaques. Arterial stenosis happens as a consequence of thrombosis which is caused due to a number of chemical, biological, biomechanical and physiological factors (Limei Cheng et al., 2019). Thrombosis is a blood clot abnormally formed inside a blood vessel, which is blocking the normal blood flow. Atherosclerosis can be formed in any parts of the arterial system but if it is formed in one of the coronary arteries, it may cause a heart attack or other associated serious health problems.

The flow in most part of coronary arteries is laminar due to the low Reynolds number $(R e)$ if there is no disturbance (For a healthy coronary artery in the resting heart working condition, Re is about 200 (Moreno and Bhaganagar, 2013)). When an atherosclerosis is formed, the flow downstream of the stenosis becomes transitional due to the instabilities created by the obstruction. The constriction inside the artery results in a jet flow, pushing the blood through the artery at higher velocity which creates recirculation zones around the jet section. Therefore, laminar flow separation and transition to turbulence, due to an increase in the velocity and the change in the fluid structure, is most likely to happen after the constricted sections. Blood flow was reported to be uniform in both internal and external carotid artery (both left and right sides of bifurcation) for the healthy artery, while changes in flow patterns were seen in the presence of a plaque inside the artery which formed reversed flow patterns along the inner wall of the diseased internal carotid artery models (Kefayati and Poepping, 2013). Flow transition to turbulence in an artery leads to a higher wall shear stress (WSS) resulting in a higher pressure drop inside the artery. Turbulence can also affect the important haemodynamic indexes such as wall shear stress as well as the location and persistence in time of recirculation regions. Therefore, it is important to predict the location of the transition to turbulence after the constrictions.

One of the parameters which triggers turbulence is the heart pulsation. Transition to turbulence in combined steady and sinusoidal oscillatory flow $\left(U=U_{m} \sin (\omega t)\right)$ for different values of Stokes'-layer-thickness ratio is displayed in Figure 1 (Lodahl et al., 1998) where $U_{m}$ is the maximum value of the sinusoidal oscillatory flow velocity at the centreline of the pipe, $R e_{c}$ is the steady Reynolds number, $R e_{\omega}$ is the oscillatory boundary-layer flow Reynolds number, $\delta$ is the Stokes layer thickness. As shown in Figure 1, for the highest value of Stokes'-layerthickness ratio $(R / \delta=3.1)$, laminar flow regime can be a valid assumption for a steady flow Re of more than 2000 and oscillatory boundary-layer flow Re above $10^{6}$. Many of the studies in the literature which investigated turbulence in a stenosed artery model assumed a steady inlet velocity profile (Biswas et al., 2016). However, turbulence inside the constricted coronary artery with the pulsatile flow is different from a model with the steady flow since the effect of the flow acceleration and deceleration on the dynamics of the flow is not considered by the steady flow assumption. Furthermore, the physiological flow is pulsatile with a complex time varying profile (Zhu et al., 2018). Therefore, turbulent structures inside the coronary artery models need to be investigated under the pulsatile flow regime. 
Another parameter which triggers the turbulent structures is the geometry of the plaques. Both shape of the plaque and the degree of stenosis, which represents the area reduction percentage, considerably affect the downstream flow behaviour. The flow characteristics such as the length of the recirculation zone and pressure losses are strongly dependent on the general shape of the stenosis (Usmani and Muralidhar, 2016). A longer recirculation zone and a higher pressure drop were reported by Young and Tsai (1973) for an asymmetric stenosis compared to the axisymmetric one with a similar degree of stenosis. Axisymmetric stenosis leads to the formation of an axisymmetric jet flow after the constricted section which is slightly deflected to the same side of the stenosis. Hence, the other side of the stenosis is a susceptible location for the plaque growth (Tambasco and Steinman, 2003). While most of the studies in the literature are focused on the axisymmetric-shaped stenosis (Ojha et al., 2006; Peterson and Plesniak, 2008; Varghese et al., 2008), an asymmetric stenosis inside an artery model has only been investigated in a few studies. Based on the considerable differences in downstream flow behaviour between the axisymmetric and asymmetric constrictions (as mentioned above) and due to the high possibility of the formation of asymmetric stenosis inside the coronary arteries (Waller, 1989), in this study the effect of the asymmetric stenosis on the flow behaviour inside the coronary artery models is investigated.

A review of the existing literature shows that the pulsatile arterial flow and the geometry of the plaque are important parameters which control the blood flow structure and its transition to turbulence. The effect of these parameters on the induced turbulence in the arterial flow is however not well understood. Hence, this study aims to investigate the transitional flow behaviour downstream of a plaque in a stenosed coronary artery with a physiological pulsatile inlet velocity. Different cases including different degrees of stenosis and a range of resting to hyperemic heart working conditions are studied. Blood flow pattern in each case is observed to develop an understanding of the flow behaviour upstream and downstream of the constriction. Turbulence kinetic energy (TKE) as an indicator of transition to turbulence and the wall shear stress is evaluated for the different cases. Furthermore, pressure drop along the stenosed artery, which represents the power required by the heart to supply the heart muscles with a uniform and constant blood flow rate, is determined. A patient specific coronary artery geometry with a blockage is investigated to discuss the applicability of the results obtained from the idealised model of the coronary artery. The results of this study will contribute to a better understanding of the changes in TKE and WSS after the asymmetric stenosis in the coronary artery models, which can be applied for determination of the susceptible locations after the stenotic region for the plaque growth by the cardiologists.

\section{Model geometry and its development}

\section{Stenosed coronary artery model}

A schematic diagram of a stenosed coronary artery and the computational domain are presented in Figure 2. In general, based on their shape the coronary arteries can be categorised into straight, curved and bifurcated. In order to develop a general understanding of the transition to turbulence in a stenosed coronary artery, instead of a patient-specific geometry of the artery 
which varies from a patient to another an idealised artery model with a circular cross section and straight length was considered in this study. To investigate the validity of the developed results for real arteries, a patient specific coronary artery model is studied. The results are discussed in the results and discussion section. The stenosed coronary artery was modelled as an asymmetric hemispherical constriction inside a rigid pipe. The assumption of rigidity of the pipe is valid since the diseases related to the blockage of the coronary artery mostly occur with ageing (as a primary risk factor of all cardiovascular diseases (Kohn et al., 2015)), and the artery wall becomes harder and stiffer with ageing (as a common disorder), and thereby loses its flexibility. Furthermore, the changes in the diameter of the coronary artery during a cardiac cycle do not change the Reynolds number in such a way that the flow behaviour changes. Consequently, the blood flow inside the coronary artery remains laminar in the presence of the changes in the artery diameter. Moreover, in a study by Torii et al. (2009), it was shown that the effects of wall compliance on time-averaged WSS and oscillatory shear index (OSI) were insignificant. Many studies in the literature have also modelled the artery wall as solid such as Zhu et al. (2018), Li et al. (2017), Foucault et al. (2017), and Plesniak and Bulusu (2016).

As shown in Figure 2, the three different degrees of stenosis were studied, including a mild stenosis, $16 \%$ area reduction, a moderate stenosis, $39.1 \%$, and a severe stenosis, $67 \%$. These three degrees of stenosis were selected to cover a wide range of coronary artery diseases from the early stage to near the final stage. Since the stenosis was modelled as an asymmetric hemispherical constriction, each degree of stenosis corresponds to a specific diameter of the hemispherical constriction shape. In other word, 16\%, $39.1 \%$ and $67 \%$ degrees of stenosis were modelled with the hemispherical diameter of $3 / 10 D, 1 / 2 D$ and $7 / 10 D$ ( $D$ is the diameter of a healthy coronary artery), respectively. Since the flow inside the artery was pulsating, the pipe length was extended from both sides (a 10-D length before and a 20-D after the stenosis section) as considered by Peterson and Plesniak (2008) to eliminate the artificial effects of the inlet and the outlet on the region of interest as well as to observe the flow behaviour, including jet flow effect, separation and recirculation zones, after the constricted section.

\section{Numerical algorithm}

ANSYS FLUENT 18.2 (ANSYS, Inc.) was employed to simulate the pulsatile blood flow inside the stenosed coronary artery model by solving the governing incompressible NavierStokes equations. The SIMPLE pressure-velocity coupling method was employed. A Womersley velocity profile (Womersley, 1955) was employed at the inlet to represent fully developed pulsatile flow (Li et al., 2017). This selection also reduces the computational cost because there is no need to use a long extended length before the inlet for development of the flow. Furthermore, as shown by Moyle et al. (2006) the choice of fully developed Womersley velocity profile at the inlet of an artery gives a good estimation of in-vivo three time-dependent components of measured velocity of an artery which is usually practically unavailable. For the momentum equations, second order upwind scheme and for the pressure discretisation, second order scheme were employed ( $\mathrm{Li}$ et al., 2017). The residual error convergence threshold was set as $10^{-5}$. A time step size of $5 \times 10^{-5} S$ was selected such that the Courant number is much 
less than unity which means that fluid particles move from cell to cell within one time step (at most). The chosen time scale results in 6,600 to 20,000 time steps per cardiac cycle (for resting and hyperemic flow conditions, respectively) which is in the same range as used by ValenSendstad and Steinman (2014). In their study, they showed that the range of high resolution time step and mesh elements provides a correct prediction of wall shear stress especially near the vulnerable regions compared to the normal resolution solutions.

In the present study, the blood was modelled as a Newtonian fluid as the diameter of the coronary artery model is about $3 \mathrm{~mm}$. According to Nichols et al. (2011), blood can be assumed as a Newtonian fluid in arteries with a diameter above $0.5 \mathrm{~mm}$ due to the high Reynolds number. Furthermore, blood is found to behave as a Newtonian fluid at shear rates greater than $100 s^{-1}$ (Jahangiri et al., 2017). As the shear rates applied to the coronary arteries are larger than 100 $s^{-1}$ (Sakariassen et al., 2015), the employed Newtonian assumption is valid. Moreover, in the arteries with diameters larger than $1 \mathrm{~mm}$, blood behaves as a single-phase homogeneous viscous fluid (Haynes, 1960). Therefore, in this study, blood was modelled as a single-phase Newtonian fluid model. The blood density, $\rho$, was set as $1060 \mathrm{~kg} / \mathrm{m}^{3}$ (Cutnell et al., 2015) and its kinematic viscosity, $\mu$, was set to 0.0034 Pa.s (Li et al., 2017).

The maximum mean and peak Reynolds numbers in coronary arteries even in hyperemic working condition are about 500 and 900, respectively (Lodahl et al., 1998). Therefore, the flow regime throughout the domain remains laminar (as recommended by Zhu et al. (2018)) and a laminar solver is able to resolve all flow details. Hence, the laminar solver was employed in this study. Four different structured grids with 205000, 421000, 640000, and 1035000 elements were employed to perform a mesh independency study and finally a fine structured grid with 640000 elements was adopted using ICEM 18.2, as shown in Figure 3. The fine mesh structure was chosen to make sure that the first layer height near the wall leads to $y^{+}$less than one to make sure that the solver is capable of solving the smallest length scales of the flow. Furthermore, the choice of structured mesh elements results in faster convergence and higher resolution comparing to the unstructured mesh elements with the same number of elements.

\section{Boundary conditions}

A physiological flow waveform corresponding to the coronary artery was employed as the velocity inlet (Davies et al., 2008), as shown in Figure 4. This waveform was modelled using the first 32 Fourier modes of Fast Fourier Transform (FFT) which accurately represented the original waveform with a maximum error of 1.4\%. A user defined function (UDF) C-file based on the waveform FFT terms was written to address the inlet condition. As shown in Figure 4, the blood flow cycle in the coronary artery is divided into two phases, systolic and diastolic. The flow rate is higher in the diastolic phase since during the systolic phase the heart muscle contracts and consequently, constricts the coronary heart vessels. During the diastolic phase, the heart muscles relax and hence, enable the blood to flow through the coronary arteries. Because of the contraction of the heart muscles during the systole, coronary blood flow rate reaches its minimum during the cardiac cycle. By relaxing the heart muscles after the contraction, the blood flow is pumped to the coronary arteries similar to a jet flow. The highest 
acceleration happens during this phase and the blood pushing continues towards its maximum velocity happening during the diastolic phase of the cardiac cycle. After that, the blood flow rate reduces to complete the cardiac cycle. The maximum deceleration happens during this part of the cardiac cycle. Four velocity and acceleration optimal time steps in the flow waveform, shown in Figure 4, were of interest in this study including the time corresponding to the maximum and minimum velocity as well as the maximum acceleration and deceleration.

The selection of outlet boundary condition has an insignificant effect on the haemodynamic behaviour. Therefore, pressure outlet with a zero-pressure boundary condition was considered in this study according to Liu et al. (2015). Furthermore, no-slip boundary condition at the walls was considered.

\section{Validation of the model}

In order to validate the numerical model of the pulsatile flow inside a constricted pipe, a comparison was made using the experimental results of Peterson and Plesniak (2008). Their work was focused on a pulsatile flow in a constricted artery model with the waveform shown in Figure 5b. Their stenosis model was axisymmetric generated by revolving an offset cosine curve about the artery axis of symmetry as follows:

$$
\begin{aligned}
& S(z)=\frac{D}{2}\left[1-s_{0}\left(1+\cos \left(\frac{2 \pi\left(z-z_{0}\right)}{L}\right)\right)\right], \\
& y=S(z) \cos \theta \\
& x=S(z) \sin \theta
\end{aligned}
$$

where $s_{0}$ is the fraction of un-occluded area and $z_{0}$ is the distance from the inception point of the stenosis to the throat. A model with the same geometry and conditions has been developed. Fully developed pulsatile pipe non-dimensionalised flow velocity at various phases including $t / T=0.003,0.167,0.278$, and 0.556 , which represented different peaks in the inlet waveform, obtained from the current simulation were compared with the experimental data of Peterson and Plesniak (2008) (Figure 5c). Furthermore, another comparison was made between the centreline velocity $\left(u_{c l}\right)$ waveforms at $2 \mathrm{D}$ upstream and downstream of the mid-stenosis section (Figure $5 \mathrm{~d}$ ). The velocity was non-dimensionalised based on the average value at the centreline of the pipe upstream of the stenosis $\left(u_{c}\right)$. The comparison showed a good agreement between their experimental results and the results of current simulation.

\section{TKE calculation}

Although there are different indicators that can be used to quantify the transition to turbulence, the turbulence kinetic energy is selected to quantify the transitional flow behaviour. The reason is the fact that turbulent kinetic energy represents the velocity fluctuations in the three directions in one parameter instead of using different parameters for different velocity directions. Furthermore, the use of turbulent kinetic energy for investigation of transitional flow behaviour of stenosed artery model (Khair et al., 2015) and aortic stenosis model (Zhu et al., 2018) has widely studied in the literature. The calculations were performed over five pulsation cycles. In order to separate small turbulences from the main pulsatile fluctuations, a phase-average technique was utilised to calculate the mean variables as: 


$$
\langle f\rangle_{p a}(x, y, z, t)=\frac{1}{N-1} \sum_{n=1}^{N-1} f(x, y, z, t+n T),
$$

where $f$ is a flow variable including velocity components in different directions and $N$ is the number of pulsation cycles $(N=5)$. The phase-averaging technique was performed over the last 4 cycles in order to eliminate the errors associated to the model initialisation. The turbulent velocity fluctuations, $f^{\prime}(x, y, z, t)$, in all directions were then calculated as

$$
f^{\prime}(x, y, z, t)=f(x, y, z, t)-\langle f\rangle_{p a}(x, y, z, t),
$$

The velocity fluctuations were calculated for four pulsation cycles from the second cycle to the fifth cycle using an in-house MATLAB code. The code was developed using the velocity components of each plane for each time step extracted from ANSYS FLUENT. Based on the velocity fluctuations, TKE was calculated by the following:

$$
T K E(x, y, z, t)=\left(\frac{1}{2}\right)\left(\overline{(\dot{u}(x, y, z, t))^{2}}+\overline{(\dot{v}(x, y, z, t))^{2}}+\overline{(\dot{w}(x, y, z, t))^{2}}\right),
$$

Area-weighted average values of TKE, $T K E_{a w}(z, t)$, on various cross-sectional planes in stream-wise direction $\left(A_{i}\right)$ were calculated from Equation (6). A total of 48 cross-sectional planes in stream-wise direction were defined with higher concentration in the vicinity of the stenosis (demonstrated in Figure 6).

$$
T K E_{a w}(z, t)=\frac{1}{A_{i}} \iint T K E(x, y, z, t) d A,
$$

Comparison of the area-weighted average TKE for different cycles, shown in Figure 7 for the max acceleration phase as an example, shows that the value of the $T K E_{a w}$ of the fifth cycle does not change compared to the fourth cycle (less than $0.7 \%$ ). Hence, all the results are reported for the fourth cycle.

\section{Results and discussion}

Five different cases in two main categories are studied including different degrees of stenosis as well as a range of resting to hyperemic heart working conditions, as given in Table 1 . The first three cases are related to the changes in the heart beat. At a typical resting condition, the heart beats once every second $(T=1)$ (case 1$)$. At this condition, the mean flow velocity inside the coronary artery is reported to be $1.1 \mathrm{ml} / \mathrm{s}$ (Grist et al., 1997). At a hyperemic condition, the heart can beat up to three times per second $(T=0.33)$ with a mean flow rate of $3.9 \mathrm{ml} / \mathrm{s}$, as reported by Grist et al. (1997) (case3). Case 2 represents the condition in between the resting and hyperemic heart working conditions with the heart beat rate of $120 \mathrm{~min}^{-1}$ and the mean flow rate of $2.5 \mathrm{ml} / \mathrm{s}$. Different degrees of stenosis are considered in case 4 and 5 representing a mild stenosis $16 \%$ (case 4 ) and a moderate stenosis (case 5) at hyperemic flow conditions. A severe stenosis is considered in all other cases (case 1 to 4 ). It is important to note that since the susceptibility of heart failure is higher at severe conditions, the highest degree of stenosis is considered for the cases with different heart working conditions while for the hyperemic condition all three degrees of stenosis (i.e. mild, moderate and severe) are studied. At the 
hyperemic condition, as mentioned earlier, the heart works harder to provide higher pressure to push the blood through the arteries with higher velocity. That is why existence of artery blockage at the hyperemic condition results in more serious problems (which is studied in this project) compared to the heart rest condition. Therefore, different degrees of stenosis were studied at the hyperemic condition.

At different heart working condition, given in Table 1, the heart beat rate is different and consequently each cardiac cycle time period $(T)$ is different. This results in changing the Womersley number, a dimensionless parameter governing the pulsatile flow, based on its definition which is:

$$
\text { Wo }=\frac{D}{2} \sqrt{\frac{2 \pi}{T} \frac{\rho}{\mu}}
$$

In this study, Womersley number for the different cases is varied because of the changes in the time period as presented in Table 1.

\section{Transitional turbulent behaviour of different phases of a pulsation cycle}

The main focus of this section is on the four different phases on the pulsation cycle representing maximum deceleration, minimum velocity, maximum acceleration, and maximum velocity, respectively, because of their importance, as explained in the previous section. Due to the large amount of data, the most physiological severe condition (i.e. hyperemic flow with $67 \%$ degree of stenosis) is studied in detail in this section. The area-weighted average of turbulent kinetic energy at different cross-sectional locations along the artery is displayed in Figure 8 for the four phases for the case with $67 \%$ degree of stenosis and hyperemic flow condition. It is worth mentioning that the turbulence generation inside the coronary artery model is not due to the reference Reynolds number, which is calculated based on the mean velocity of the flow and the pipe diameter. Although the mean upstream Reynolds number is relatively low inside the artery (it is about 500 for hyperemic flow condition), the blockage effect due to the stenosis inside the coronary artery creates transitional flow behaviour, which is evidenced by the TKE profile. The mean local Reynolds number at the location of blockage can increase up to about three times for the case with $67 \%$ degree of stenosis. Furthermore, the peak velocity during a cardiac cycle, as shown in Figure 4, is about two times higher than the mean velocity that results in the peak Reynolds number about twice as the mean Reynolds number. Hence, the local temporal Reynolds number can increase up to about six times as the mean upstream Reynolds number that results in periodic transition to turbulence during a cardiac cycle. This trend is consistent with the studies in the literature such as Khair et al. (2015) and Zhu et al. (2018), as explained in the introduction section. The TKE profile shows several peaks which are related to the effect of the constriction on the flow. The first noticeable jump occurs immediately after the stenosis for all the phases. This jump in the turbulent kinetic energy is related to the jet effect formed due to the reduction in the flow cross section which causes a considerable increase in the blood velocity. For example, for the case with $67 \%$ degree of stenosis, based on the continuity equation, the mean velocity can increase up to three times 
immediately after the stenosis compared to the upstream mean velocity. This causes a significant shear between the jet flow and the surrounding flow leading to a sudden jump in the TKE profile. Furthermore, this creates intense vortices in the post-stenotic region. The largest jump occurs at the maximum velocity phase at which TKE is significantly higher than the other phases. The minimum velocity phase creates the weakest jet flow effect which is accompanied with the lowest rise in the TKE profile after the stenosis. As the jet flow effects slightly disappear, the turbulent structures grow to reach their maximum intensity at around 5D downstream of the stenosis. This causes the second jump in the TKE profile which for all phases happen at about $z / D=5$. The highest TKE in this phase is related to the maximum velocity phase as the higher jet flow velocity in this phase induces a larger shear layer between the jet flow and the surrounding flow which consequently, increases the velocity fluctuations. However, the lowest TKE at this phase is related to the maximum acceleration phase despite the larger mean velocity compared to the other two cases. In the maximum acceleration phase, the largest acceleration means the largest force on the bulk of the fluid which can result in suppressing the turbulence structures and consequently, reducing TKE. In the deceleration phase, the heart decelerates the blood flow inside the coronary artery which helps the eddies to grow faster. That is why, after the maximum velocity phase, the maximum deceleration phase owns the highest TKE. In the far downstream of the stenosis, all the turbulent structures are vanished and flow re-laminarises. This trend is completely in agreement with the results reported by Khair et al. (2015) where re-laminarisation happens downstream of the stenosis because the turbulence is damped about 10D downstream of the stenotic region.

Figure 9 displays the instantaneous coherent structures identified by the lambda- 2 criterion coloured by the stream-wise velocity at four phases in the fourth pulsation cycle of the case3. Lambda-2 criterion is a detection algorithm that can identify vortices in a 3D fluid velocity field (Jeong and Hussain, 2006). The longest vortex structures are related to the maximum acceleration case (Figure 9c) where the highest forces due to the highest acceleration create these longitudinal structures and prevent the formation of the turbulent structures in this phase after the blockage. This clearly supports the lowest value of the turbulent kinetic energy for the maximum acceleration phase, as presented in Figure 8. There is a general trend of the formation of quite long vortices after the stenotic region in the other phases, which is due to the jet flow effect, as can be observed in Figure 9a, b and d. Further downstream of the stenotic region, the turbulent structures grow creating disturbances in the flow regime in the form of eddies in the flow. Far downstream of the stenosis and near the artery model outlet, the turbulent structures dissipate and the flow becomes relaminarised. The trends are governed for the other phases as well. The turbulent structures have their highest intensity for the maximum velocity phase and after that maximum deceleration phase has more intense turbulences compared to the minimum velocity phase. Therefore, the turbulence structures shown in Figure 9 are in agreement with the variations of TKE given in Figure 8.

Velocity profiles along different lateral lines ( $x=0$ and $-D / 2 \leq y \leq D / 2)$ downstream of the stenotic region up to $10 \mathrm{D}$ at four phases in the fourth pulsation cycle of the case 3 are shown in Figure 10. The general trend in the velocity profile in different cross-sectional planes is that 
the maximum velocity belongs to the maximum velocity phase and the minimum is for the minimum velocity phase, as expected. However, there are some exceptions. At the beginning stage of the jet flow immediately after the stenosis region, $(z / D=1)$, the maximum velocity is mostly related to the maximum acceleration phase except a small region near the core of the artery model where the maximum velocity phase has the maximum velocity. The jet flow grows past the stenotic region which is more significant in the maximum velocity phase. This increasing trend continues up to 3D downstream of the stenosis where the maximum velocity of the jet flow occurs at the centre of the artery model for the maximum velocity phase which is about $3.5 \mathrm{~m} / \mathrm{s}$. The largest value of the shear layer between the jet flow and the surrounding blood flow near the wall occurs in this location. After that, the jet flow effect is gradually reduced in the stream-wise direction. The jet flow effect is almost vanished at $8 \mathrm{D}$ downstream of the stenotic region. After this location, a sudden reduction in the central velocity of the maximum velocity phase is observed $(z / D=9)$ which is related to the positive pressure gradient that is pushing the fluid back. The near wall velocity trend is to some extent different from the above-described behaviour. The near wall flow regime is separated from the core jet flow regime by the induced shear layers due to the high central velocity. In the developing jet flow regime, starting from the early post-stenotic region and continuing up to 3D downstream of the stenosis, the reverse flow between the artery wall and the shear layers is intensified for all phases. Past the location with maximum jet flow effect, the reverse flow reduces gradually along with the jet velocity reduction. The reverse flow is completely vanished at about 8D downstream of the stenosis. It is interesting to note that the higher length of the jet flow, the higher length of the reverse flow for almost all phases. For example, as can be seen in the velocity profile at $z / D=3$, the highest velocity value of the reverse flow is related to the maximum velocity phase which has the highest core flow velocity. This trend is not valid at the maximum acceleration phase. As explained earlier, in this phase the heart pushes the blood back in the stream-wise direction with its highest acceleration during the cardiac cycle. Therefore, there is no reverse flow during this phase of the cardiac cycle at any stream-wise locations.

Static pressure distribution along the stream-wise direction at four phases in the fourth pulsation cycle of the case3 is displayed in Figure 11. Pressure drop is an important parameter since it represents the power required by the heart to supply the heart muscles with a uniform and constant blood flow rate. Higher pressure drop means higher heart muscle effort to provide the same blood flow rate for the organs to work properly. In order to perform a comparison between the four phases during the cardiac cycle, a zero gauge pressure is considered in the outlet of the artery model (as explained in the boundary condition section, selection of outlet boundary condition has almost insignificant effect on the haemodynamic results (Liu et al., 2015)). The pressure drop across the stenosis is found to vary significantly during the systole and diastole which is in agreement with that reported by (Li et al., 2009). The maximum pressure difference between the upstream and downstream of the stenosis is related to the maximum acceleration phase. High acceleration means that large forces are exerted to the flow and consequently, the high pressure differences illustrate the high pressure gradient which is 
also the reason of the lack of reverse flow in this case. At this phase, as explained before, the blood is pushed through the artery with the highest acceleration during the cardiac cycle and therefore, this creates the highest pressure drop during the cardiac cycle. The minimum pressure difference is related to the minimum velocity phase. For an artery model with a $67 \%$ degree stenosis during the hyperemic flow condition, the maximum pressure difference, which is associated with the maximum acceleration phase, is about $5800 \mathrm{~Pa}$ that requires the heart to work harder. Furthermore, the minimum pressure difference, about $280 \mathrm{~Pa}$, happens when the flow velocity is minimum. In the maximum deceleration phase, the heart decelerates the blood flow in the artery consequently creating negative back pressure. The negative back pressure for an artery model with a 67\% degree of stenosis during the heart activating mode is about 2700 Pa which occurs far upstream of the stenosis. In all phases during a cardiac cycle, a sudden drop in the pressure distribution profile along the stream-wise direction can be seen which is about a sudden increase in the velocity due to the cross section reduction in the stenosis location. The value of this sudden drop in the pressure is directly related to the value of the jump in the stream-wise velocity where the highest is related to the maximum velocity phase and the lowest is related to the minimum velocity phase. After the stenosis, for both maximum and minimum velocity phases, there is a negative pressure zone spreading from the stenotic region up to $6 \mathrm{D}$ downstream of the stenosis. Past the stenotic region all the phases of a cardiac cycle retrieve the pressure changes and reach towards the zero gauge pressure at the outlet of the artery model.

Stream-wise distributions of the wall shear stress along the top line (the same side of the stenosis), the side line and the bottom line (the opposite side of the stenosis) at four phases in the fourth pulsation cycle of the case 3 are shown in Figure 12. For all line locations (shown in Figure 12a), the maximum wall shear stress happens in the mid-stenosis region. At this location, because of the cross section reduction and the consequent increase of mean velocity, larger shear forces are applied to the walls creating higher wall shear stresses. Far downstream and upstream of the stenotic region near to the inlet and outlet of the model, the value of wall shear stress is considerably small for all phases. The highest wall shear stress is related to the maximum velocity phase which is about $10 \mathrm{~Pa}$ while the lowest, about $1 \mathrm{~Pa}$, is related to the minimum velocity phase. Furthermore, the highest wall shear stress in the cardiac cycle is related to the maximum velocity phase and the lowest is for minimum velocity phase. Since the blood force on the stenosis wall is applied to the smaller area, because of the stenosis, compared to the side and bottom regions, it results in larger wall shear stresses in the midstenosis part, as can be seen in Figure 12b. Wall shear stress at the sides of the stenosis remains positive in the stream-wise direction except small regions before and after the stenosis. This means that two small separation regions exist before and after the stenosis. After the stenosis, wall shear stress at the same side of the stenosis in the stream-wise direction increases gradually to reach its maximum value around 5D downstream of the stenotic region. This trend is valid for all the cardiac cycle phases. However, the corresponding value for the maximum velocity phase is much higher than the other phases. The location of maximum wall shear stress at the downstream of the stenosis is the same as the region with a jump in the TKE profile as 
explained in Figure 8. The turbulent structures are intensified after the jet-affected region and create considerable wall shear stresses downstream of the stenosis in the same side of the stenosis. The higher shear stress results in the higher possibility of the plaque rupture. Because of the considerable large wall shear stresses downstream of the stenosis at the same side of it, there is almost no possibility for the formation of the second plaque there. Far downstream of the stenosis where the turbulence fades, wall shear stress reduces gradually reaching a constant value which is due to the pressure exerted to the wall from the blood flow. Large recirculation zones can be observed downstream of the stenotic region in the side and bottom of the stenosis which is shown in Figure 10c and 10d by the zones with negative wall shear stresses. The separation starts after the stenosis in the side part of the stenosis for all cardiac phases (except the maximum acceleration phase) and flow reattaches to the wall far downstream of the stenosis $(z / D=10)$. The flow separation is postponed after the stenosis to about 5D downstream of the stenosis in the opposite side of the stenosis and attached to the wall 5D after the separation. Therefore, the region between 5D and 10D after the stenosis, at the side and bottom regions of the first stenosis, is the location with the high possibility of the formation of the second stenosis due to the existence of the recirculation zones. There is no negative wall shear stresses, no flow separation, on the wall for the maximum acceleration phase which is supporting the previous results explained in Figure 10 and 11. It is important to note that the coronary stenosis is more likely to form in the locations with disturbed flow conditions which is characterised by low WSS (Zhang et al., 2015). Furthermore, low WSS appears to be the most important hemodynamic parameter promoting atherosclerotic plaque growth that in turn influences the blood flow and WSS distribution (Arzani, 2020).

As the highest wall shear stress during a cardiac cycle happens during the maximum velocity phase, as explained before, wall shear stress contour of this phase for the fourth pulsation cycle of the case 3 from both top and bottom views is displayed in Figure 13. Hence, the maximum velocity phase will be selected for further analysis in the rest of the results. As explained, the maximum wall shear stress occurs at the mid-stenosis location which is highlighted in dark red in the top view and orange in the bottom view. The dark blue region, mainly distributed after the stenosis in the side and bottom parts of the stenosis, represents the separated zones due to their negative values of wall shear stress.

In order to be more accurate in finding the susceptible locations for flow separation based on the wall shear stresses during a cardiac cycle, the stream-wise distributions of the wall shear stress at different lateral and vertical positions of maximum velocity phase is illustrated in Figure 14. The highest shear stress is slightly reduced from the top stenosis region (the same side of the stenosis) to the opposite side. This means that the highest wall shear stress at the mid-stenosis cross section happens in the top side of the stenosis and the lowest happens in the opposite side of it. A small recirculation zone can be seen immediately before and after the stenosis at only the top region of the bump and close to it (line a-b). For these two positions, no more separation occurs in the downstream of the stenosis. Separation starts from the side of the stenosis (line c) and continues up to the opposite side of it (line e). Moving from the side to the opposite region of the stenosis, separation is postponed to the far downstream of the 
stenosis. For example, at the side of stenosis (line c), separation starts $1 \mathrm{D}$ after the stenosis which is moved downstream to the 4D for line $\mathrm{d}$ and finally 6D downstream of the stenosis for the opposite region of the stenosis (line e). Based on the results, downstream of the stenosis in the top stenosis region and close to it, there is no possibility for the formation of the second stenosis. However, the possibility of the formation of the second stenosis downstream of the first stenosis increases in the side region of the stenosis up to the opposite region.

More detailed instantaneous velocity contours and vectors at different vertical, lateral and circular cross-sectional planes in stream-wise direction of the maximum velocity phase are shown in Figure 15. The jet flow effect starts at the location of stenosis and therefore, the flow reaches its maximum velocity (about $3.5 \mathrm{~m} / \mathrm{s}$ ) at this location at the vertical direction (Figure 15a). The jet flow effect extends up to about 5D downstream of it. The disturbances in the velocity patterns grow after dissipation of the jet effect. It creates turbulent structures in the downstream flow which mainly occur from 5D to 10D downstream of the stenotic region (as displayed in Figure 15a and 15b). After that the flow starts to re-laminarise which is completed far downstream of the stenosis near the outlet of the artery model (18D downstream). This means that the velocity profiles regain their parabolic shape similar to the upstream of the stenosis (as demonstrated in Figure 15a and 15b). Two symmetric vortex tubes are formed around the jet flow immediately after the stenosis extending to 5D downstream of the stenotic region which is highlighted in dark blue in Figure 15c. This region with the negative velocity value (displayed in dark blue with about $-0.53 \mathrm{~m} / \mathrm{s}$ ) is the separated region as a consequence of the adverse pressure gradient. Based on the results of Figure 15, separation is most likely to happen at the side of the stenosis (Figure 15b) and there is almost no possibility for the flow separation at the same side of the stenosis (Figure 15a). Furthermore, a small recirculation zone can be observed far downstream of the stenosis at the bottom side of it, starting from 5D and finishing at about 10D downstream of the stenotic region (Figure 15a).

\section{Effect of heart working condition and degree of stenosis}

In this section, the effects of the degree of stenosis and the heart working conditions for a range of resting to hyperemic conditions are investigated. The area-weighted average of TKE along different cross-sectional planes at the maximum velocity phase of the fourth pulsation cycle for different heart working conditions and degrees of stenosis are shown in Figure 16a and 16b, respectively. As shown in Figure 16a, as the heart works harder to provide higher flow rate for different organs, the jump in the TKE profile due to the jet flow effect at the mid-stenosis location is increased significantly, as the corresponding value for the resting condition is 0.05 $(\mathrm{m} / \mathrm{s})^{2}$ and for hyperemic condition is $0.6(\mathrm{~m} / \mathrm{s})^{2}$ (more than one order of magnitude larger). It is interesting to note that there is almost no jump in the TKE profile for the resting heart working condition, even for the artery model with $67 \%$ degree of stenosis which means that no turbulent structures are formed even after the stenotic region with high degree of stenosis. On the other hand, another jump which is related to the formation of turbulence structures downstream of the stenosis exists for the moderate heart working condition (case 2) and hyperemic one (case 3). For the hyperemic flow condition, the jump in the area-weighted 
average turbulent kinetic energy profile due to the formation of turbulences occurs at $5 \mathrm{D}$ downstream of the stenotic region and its value is about $0.4(\mathrm{~m} / \mathrm{s})^{2}$. Whereas, the jump in the TKE profile for the moderate heart working condition (case 2), about $0.13(\mathrm{~m} / \mathrm{s})^{2}$, occurs at around 7D downstream of the stenosis. This means that the location where the intensity of the turbulent structures is the highest shifts towards the stenosis for the conditions in which the heart is working harder (hyperemic flow condition) and the turbulence kinetic energy and intensity are much larger in hyperemic flow conditions compare to the others. Since in the hyperemic heart working condition, the heart is required to pump more blood to the arteries and the blood flow rate is larger, the Reynolds number inside the arteries also increases considerably. This finding is in agreement with the results of (Khair et al., 2015; Biswas et al., 2016) reporting that for the higher values of Reynolds number, the transition locations, (the sudden jump in the TKE profile), moved closer to the constricted location.

According to Figure 16(b), TKE at the mid stenosis region is larger for the higher the degree of stenosis due to the increased jet flow effect. As for the higher degree of stenosis the cross sectional area of the artery is reduced, and based on the continuity equation, the blood mean velocity increases which consequently leads to increase in the jet flow effect. No turbulence, based on the TKE profile, can be observed in the artery model with the low degree of stenosis (16\%). However, by increasing the degree of stenosis, a jump in the TKE profile which is related to the formation of turbulent structures in the downstream of the stenosis is observed. The higher the degree of stenosis, the higher is the value of TKE and the level of the turbulence downstream of the artery. For the $39.1 \%$ degree of stenosis, the jump is located at $7 \mathrm{D}$ downstream of the stenosis with a corresponding TKE value of about $0.1(\mathrm{~m} / \mathrm{s})^{2}$. For the higher degree of stenosis case (67\%), the peak location shifts toward the stenosis (5D) and its value is increased to $0.6(\mathrm{~m} / \mathrm{s})^{2}$. Therefore, the jump in the TKE profile moves closer to the stenosis for the higher degrees of stenosis. The obtained results about the effect of degree of stenosis on the location of the jump in the TKE profile as well as its corresponding value are strongly supported by the literature (Khair et al., 2015; Biswas et al., 2016). Table 2 summarises the maximum values of area-weighted average of TKE for different cases considered in this study based on the results explained in Figures 8 and 16. This table shows that the second and fifth cases have almost the same TKE values for different phases. This means that having a moderate stenosis degree in hyperemic heart flow condition will create the same turbulences as having a severe stenosis degree with the heart flow condition in between resting and hyperemic conditions.

The static pressure distribution along the stream-wise line located at $\mathrm{y}=-1 \mathrm{~mm}$ at $\max$ velocity phase for different heart working conditions ranging from resting to hyperemic and degrees of stenosis are shown in Figure 17(a-b). At the hyperemic heart working condition, in which the arterial system requires more blood flow rate, the value of the pressure drop across the stenosis in the artery model is larger compared to the resting heart working condition which forces the heart to work faster to compensate the pressure drop. The pressure drop in the resting condition is about $700 \mathrm{~Pa}$, in the moderate heart working condition is about $2300 \mathrm{~Pa}$ and finally in the hyperemic heart working condition is about $4700 \mathrm{~Pa}$ (about twice of the moderate heart 
working condition and 6 times more than the resting heart working condition). A sudden pressure drop can be observed in all heart working conditions immediately after the stenosis which occurs due to an increase in the velocity which can be explained based on the Bernoulli equation stating that with an increase in the fluid velocity, a decrease in the pressure occurs simultaneously. Furthermore, a region with negative pressure exists for the moderate and hyperemic heart working conditions immediately downstream of the stenosis. At this location, the hyperemic condition leads to a more negative pressure compared to the moderate heart working condition. This implies the existence of recirculation zones with higher pressure gradients after the stenosis in hyperemic heart working condition compared to the other conditions as shown in Figure 15b and 15c.

The higher degree of stenosis results in a larger pressure drop along the stenotic region in the artery (Figure 17b). The pressure drop is about $1200 \mathrm{~Pa}$ and $1600 \mathrm{~Pa}$ for the $16 \%$ and 39.1\% degrees of stenosis, respectively. It increase significantly up to $4700 \mathrm{~Pa}$ for the $67 \%$ degree of stenosis. The increase of pressure drop with the increase in the degree of stenosis is due to the reduction of the cross sectional area and increase of mean velocity as explained above. The observed trend is in agreement with the results reported by Young et al. (1975) and Flanigan et al. (1977) illustrating a remarkable jump in the pressure drop for the severe degrees of stenosis compared to the early stage stenoses. Furthermore, the same results were reported by Rotman et al. (2017) which found that the pressure drop increases exponentially as the degree of stenosis increases.

Figure 18 presents the stream-wise distributions of wall shear stress along the top, side and bottom lines at the maximum velocity phase for different heart working conditions (left column of Figure 18) and different degrees of stenosis (right column of Figure 18). The maximum wall shear stress occurs at the mid-stenosis location for all directions, as explained before, and its maximum value belongs to the hyperemic flow condition. The possibility of the formation of the second plaque for the resting heart working condition is too low except at two small regions immediately before and after the stenosis at the same side of it. Even at these locations, the recirculation zones may change the shape of the first stenosis without any chances for the formation of the second one since they are connected to the first stenosis. According to Figure 18a (left side), a small jump in the value of the wall shear stress downstream of the stenosis at the same side of it (top line) is observed which is more intense for the hyperemic heart working condition (case 3). As explained in the previous section, the separation starts downstream of the stenosis on the lateral areas of the wall (side line) as well as its bottom side (bottom line) for all heart working conditions except the resting condition. The separation and attachment region of the moderate and hyperemic heart working condition at the side line of the stenosis starts $1 \mathrm{D}$ after the stenosis and ends at about 7D downstream of the flow, respectively. The recirculation zones for the opposite side of the stenosis are moved to the downstream of the flow, i.e. the separation starts at about 6D and attaches to the wall at around 10D downstream of the stenotic region. This means that separation starts from the side of the stenosis and spreads to the bottom side of it further downstream. The magnitude of the wall shear stress is higher in 
the recirculation zone of the hyperemic heart working condition compared to the moderate and resting conditions.

According to Figure 18 (the right column), the wall shear stress at all sides of the mid-stenosis location is larger for the higher degrees of stenosis, which is due to the large vertical gradient of stream-wise velocity. No flow separation occurs at any sides of the low degree of stenosis (mild stenosis model), except a small region before and after the stenosis at the same side of it. Almost the same trend exists for the moderate stenosis model (39.1\%). It can be concluded that there is no separation in the post-stenotic region for the cases with low to moderate stenosis model. However, for the severe stenosis case the trend is different. As explained earlier, the separation occurs by increasing the degree of stenosis to severe stenosis model (67\%). The recirculation zone observed in this case has almost the same trend as the one reported by Khair et al. (2015) in terms of the separation and attachment locations.

\section{A case study: blockage inside a patient specific coronary artery model}

In this section, to demonstrate the validity of the developed results for an idealised coronary artery model to real cases and since atherosclerosis is highly influenced by the artery geometry (Doutel et al., 2019), a patient specific coronary artery geometry is studied and the flow features, wall shear stress and TKE in a stenosed patient specific coronary artery model are investigated. The results are presented for the hyperemic flow condition case with three beats per second as a cardiac cycle time period and at a flow rate of $3.9 \mathrm{ml} / \mathrm{s}$. A patient specific left anterior descending coronary artery is shown in Figure 19. The inlet geometry of patient specific coronary artery model is in the shape of an ellipse and its cross sectional area is 2.225 $\mathrm{mm}^{2}$. The patient specific artery has a degree of stenosis of $57 \%$, as shown in the dashed area in Figure 19. The cross sectional area of the artery after the blockage section expands to a crosssectional area of $4.69 \mathrm{~mm}^{2}$, which is more than twice the inlet area of the artery. For the patient specific geometry case, since the cross section of the artery is not circular, the application of Womersley velocity profile at the inlet is not valid. The reason is that the application of the parabolic or Womersley velocity profiles is limited to the problems with a circular crosssection, representing the fully developed flow regime at the inlet. Hence, in this case study, a blunt spatial velocity profile with the hyperemic flow rate, and a pulsatile temporal velocity profile were employed as an inlet boundary condition (Figure 4). To eliminate inlet disturbances and high wall shear stresses zone at the inlet, the inlet cross section of the artery was extended for 5D, as shown in Figure 19. Outlet boundary condition, flow solver, type of working fluid and its physical properties were considered identical to those of the idealised coronary artery model, as explained in the previous section. A structured mesh grid with $1,383,000$ mesh elements was generated using ICEM 18.2. Time-step was set to $5 \times 10^{-6} s$ that resulted in 66,000 time-steps per cardiac cycle. A total of 35 cross-sectional planes in stream-wise direction were defined with a higher concentration in the vicinity of the stenosis and after it to illustrate the velocity contours and TKE values.

Figure 20 displays the velocity contours at different cross-sectional planes for different phases during the third cardiac cycle. As discussed in Figure 10 and similar to the findings for the 
idealised geometry, the highest velocity is related to the maximum velocity phase during a cardiac cycle, while the lowest is related to the minimum velocity phase. The velocity contours near the inlet of the artery (inflow zone) for all phases show a uniform structure which demonstrates that the application of the inlet extension eliminates the inlet effects on wall shear stress distribution. The highest velocity for all phases occurs at the location of blockage, as expected, due to the reduction in the cross section of the artery that results in the formation of the jet flow regime after the blockage section (jet affected zone). The jet flow regime continues downstream of the blockage section about 12 planes from the stenosis location (about $0.05 \mathrm{~m}$ after the blockage section) and deflects to the bottom side of the artery due to the effect of the curvature in the patient specific artery at the location of blockage. In the far wake and after dissipation of the jet flow energy, the disturbances in the velocity contours appear that result in growth of the turbulent structures. This behaviour is consistent with the turbulence structures reported for the idealised artery geometry. Further downstream, the flow relaminarises near the outlet of the patient specific artery model (outflow zone).

Figure 21 shows wall shear stress contours for different phases of the third pulsation cycle for the hyperemic flow conditions. The highest wall shear stress range is related to the phase with a maximum velocity during the cardiac cycle. The highest wall shear stress occurs at the midstenosis location for all phases which is related to the induced large shear layer due to the formation of jet regime when the flow passes through the blockage area. As explained in the previous paragraph, the jet is deflected to the outer region of the curvature after the blockage section. This results in a lower wall shear stress zone at the inner region of the curvature (same side of the stenosis) and a higher wall shear stress zone at the outer region (opposite side of the stenosis). This is slightly different from what was found for the idealised coronary artery model. The reason is the existence of the curvature in the patient specific coronary artery geometry, which did not exist in the idealised model. This curvature changes the direction of the jet flow after the stenosis section.

The area-weighted average of TKE at different cross-sectional planes along the patient specific coronary artery is displayed in Figure 22 for the four phases during a cardiac cycle and for the hyperemic flow conditions. In order to calculate TKE for this case, the simulation was performed for five cardiac cycles, which resulted in 330,000 time-steps. The values of phaseaveraged TKE for different cycles were compared and it was shown that the area-weighted average of TKE for all cross-sectional planes are almost the same for the fourth and fifth cardiac cycles (less than $0.5 \%$ difference), which is the same as the trend explained in the TKE calculation section for the idealised coronary artery model. The results of the fourth cycle are presented in Figure 22. The distance from the inlet, shown on the primary horizontal axis, was calculated using the distance between the centres of two consecutive planes. On the secondary horizontal axis in Figure 22, the number of cross-sectional planes in stream-wise direction is shown, starting from the inlet of the artery model as shown in Figure 19, where 0 and 36 refer to the inlet and outlet of the patient specific coronary artery model, respectively. The results show that slightly after the location of blockage, TKE reaches to its highest value for all phases, which is in agreement with the findings for the idealised model, as shown in Figure 8. At this 
location, the highest TKE is related to the phase with the highest velocity, i.e. maximum velocity phase and the lowest TKE is related to the minimum velocity phase, which can be explained based on the formation of the jet flow regime after the blockage, as discussed for the idealised model. There are two more regions in the patient specific artery model with high values of TKE before the flow re-laminarises. These regions are related to the two curved sections in the geometry model; near the blockage and further downstream, as displayed in Figure 21a. These high TKE regions, due to curvature, are in agreement with the reported high values of TKE at the location of the curvature of aorta reported by Zhu et al. (2018). This phenomenon, is different from what was found earlier for the idealised coronary artery model as the idealised geometry does not include a curved section. It is worth mentioning that there is almost no turbulences at the inflow zone for all phases and the flow relaminarises downstream of the blockage section near the outlet of the geometry model (outflow zone).

\section{Conclusion}

In this study, the pulsatile blood flow inside a set of stenosed coronary arteries was modelled and the effect of different asymmetric degrees of stenosis as well as different heart working conditions on the flow patterns and hemodynamic parameters, including pressure drop and wall shear stress, were investigated. Furthermore, area-weighted average TKE along the streamwise direction was calculated to identify the transition from laminar to turbulent flow after the stenotic region. The computational model was validated against the published results in the literature.

A jump in the TKE profile was observed due to the formation of the turbulence strictures downstream of the stenosis starting from 5D downstream, where the jet flow effect due to cross section reduction is faded, to about $10 \mathrm{D}$, where the blood flow starts re-laminarisation. The location of the jump in TKE was found to be a function of the degree of stenosis and the heart working condition. It moves closer to the stenotic region by increasing the degree of stenosis as well as for the hyperemic flow condition. This region was accompanied with negative wall shear stress which shows the flow separation from the wall and formation of the recirculation zones which are susceptible locations for the formation of the second stenosis downstream of the first one. Furthermore, the results showed that based on the obtained wall shear stress results, the possible location of the second stenosis is not at the same side of the first one. The regions which are most susceptible to second stenosis are located at the opposite side of the first stenosis or at the side part of it both at downstream of it. The results of this study will assist cardiologists for determination of the susceptible locations after the stenotic region for the plaque growth.

For the severe stenosis model with the high degrees of stenosis, the heart needs to work harder to provide the same blood flow inside the arteries. Furthermore, the peak location of TKE due to turbulences moved closer to the stenosis at this condition. The same trend was also seen for the hyperemic heart working condition. Based on the obtained results, for the more intense situations both in terms of degrees of stenosis and heart working conditions, the closer region after the first stenosis should be considered in terms of the possibility of the formation of the 
second stenosis. When the degree of stenosis is high, the heart needs to work harder and based on the results, the turbulences and recirculation zones get closer to the stenosis and increase the chance of the growth of the stenosis. However, it is interesting that for the high degree of stenosis case, there was no separation occurred downstream of the stenosis for the resting to moderate heart working condition. This clearly means that one way to have a medically stable condition for a patient suffering from high degrees of atherosclerosis is to prevent them from hyperaemia.

The obtained results of this study were found to be applicable for the patient specific coronary artery geometry. Since the patient specific arteries cannot exactly be classified as straight or curved arteries, there are some differences between the results of idealised straight artery model with the real geometry one. However, the trends, shown in results and discussion section, prove the applicability of the developed results for patient specific geometries, although there are some minor differences. The peak in the wall shear stress distribution and area-weighted average TKE is shown at the location of blockage of the patient specific coronary artery model that is the same as behaviour observed for an idealised geometry.

\section{Acknowledgement}

Financial support for the project has been provided by the Australian Government Research Training Program Scholarship. This work was supported with supercomputing resources provided by the Phoenix HPC service at the University of Adelaide.

\section{References}

Arzani, A. 2020. Coronary artery plaque growth: A two-way coupled shear stress-driven model, Int J Numer Method Biomed Eng, 36: e3293.

Biswas, D., Casey, D. M., Crowder, D. C., Steinman, D. A., Yun, Y. H., and Loth, F. 2016. Characterization of transition to turbulence for blood in a straight pipe under steady flow conditions, Journal of Biomechanical Engineering, 138: 1-12.

Cutnell, J. D., Johnson, K. W., Young, D., and Stadler, S. 2015. Physics, 10th edition, Wiley.

Davies, J. E., Parker, K. H., Francis, D. P., Hughes, A. D., and Mayet, J. 2008. What is the role of the aorta in directing coronary blood flow?, Heart, 94: 1545-47.

Doutel, E., Viriato, N., Carneiro, J., Campos, J., and Miranda, J. M. 2019. Geometrical effects in the hemodynamics of stenotic and non-stenotic left coronary arteries-numerical and in vitro approaches, Int J Numer Method Biomed Eng, 35: e3207.

Flanigan, D. P., Tullis, J. P., Streeter, V. L., Whitehouse, W. M., Jr., Fry, W. J., and Stanley, J. C. 1977. Multiple subcritical arterial stenoses: Effect on poststenotic pressure and flow, Annals of Surgery, 186: 663-8.

Foucault, E., Huberson, S., Braud, P., and Coisne, D. 2017. On the pulsatile flow through a coronary bifurcation with stent, European Journal of Mechanics - B/Fluids, 61: 17786.

Grist, T. M., Polzin, J. A., Bianco, J. A., Foo, T. K., Bernstein, M. A., and Mistretta, C. M. 1997. Measurement of coronary blood flow and flow reserve using magnetic resonance imaging, Cardiology, 88: 80-9. 
Haynes, R. H. 1960. Physical basis of the dependence of blood viscosity on tube radius, American Journal of Physiology, 198: 1193-200.

Jahangiri, M., Saghafian, M., and Sadeghi, M. R. 2017. Numerical simulation of nonnewtonian models effect on hemodynamic factors of pulsatile blood flow in elastic stenosed artery, Journal of Mechanical Science and Technology, 31: 1003-13.

Jeong, J., and Hussain, F. 2006. On the identification of a vortex, Journal of Fluid Mechanics, 285: 69-94.

Kefayati, S., and Poepping, T. L. 2013. Transitional flow analysis in the carotid artery bifurcation by proper orthogonal decomposition and particle image velocimetry, Medical Engineering \& Physics, 35: 898-909.

Khair, A., Wang, B.-C., and Kuhn, D. C. S. 2015. Study of laminar-turbulent flow transition under pulsatile conditions in a constricted channel, International Journal of Computational Fluid Dynamics, 29: 447-63.

Kohn, J. C., Lampi, M. C., and Reinhart-King, C. A. 2015. Age-related vascular stiffening: Causes and consequences, Frontiers in Genetics, 6: 112.

Li, S., Chin, C., Thondapu, V., Poon, E. K. W., Monty, J. P., Li, Y., Ooi, A. S. H., Tu, S., and Barlis, P. 2017. Numerical and experimental investigations of the flow-pressure relation in multiple sequential stenoses coronary artery, Int J Cardiovasc Imaging, 33: 1083-88.

Li, Z. Y., Taviani, V., Tang, T., Sadat, U., Young, V., Patterson, A., Graves, M., and Gillard, J. H. 2009. The mechanical triggers of plaque rupture: Shear stress vs pressure gradient, The British Journal of Radiology, 82 Spec No 1: S39-45.

Limei Cheng, Guo-Wei Wei, and Leil, T. 2019. Review of quantitative systems pharmacological modeling of thrombosis, Communications in Information and Systems, 19: 219-40.

Liu, B., Zheng, J., Bach, R., and Tang, D. 2015. Influence of model boundary conditions on blood flow patterns in a patient specific stenotic right coronary artery, BioMedical Engineering OnLine, 14: 6-23.

Lodahl, C. R., Sumer, B. M., and FredsØE, J. 1998. Turbulent combined oscillatory flow and current in a pipe, Journal of Fluid Mechanics, 373: 313-48.

Moreno, C., and Bhaganagar, K. 2013. Modeling of stenotic coronary artery and implications of plaque morphology on blood flow, Modelling and Simulation in Engineering, 2013: 14.

Moyle, K. R., Antiga, L., and Steinman, D. A. 2006. Inlet conditions for image-based cfd models of the carotid bifurcation: Is it reasonable to assume fully developed flow?, $J$ Biomech Eng, 128: 371-9.

Nichols, W., O'Rourke, M., and Vlachopoulos, C. 2011. Mcdonald's blood flow in arteries, sixth edition: Theoretical, experimental and clinical principles, CRC Press.

Ojha, M., Cobbold, R. S. C., Johnston, K. W., and Hummel, R. L. 2006. Pulsatile flow through constricted tubes: An experimental investigation using photochromic tracer methods, Journal of Fluid Mechanics, 203: 173-97.

Peterson, S. D., and Plesniak, M. W. 2008. The influence of inlet velocity profile and secondary flow on pulsatile flow in a model artery with stenosis, Journal of Fluid Mechanics, 616: 263-301. 
Plesniak, M. W., and Bulusu, K. V. 2016. Morphology of secondary flows in a curved pipe with pulsatile inflow, Journal of Fluids Engineering, 138: 101203.

Rotman, O. M., Zaretsky, U., Shitzer, A., and Einav, S. 2017. Pressure drop and arterial compliance - two arterial parameters in one measurement, Journal of Biomechanics, 50: 130-37.

Sakariassen, K. S., Orning, L., and Turitto, V. T. 2015. The impact of blood shear rate on arterial thrombus formation, Future Science OA, 1: 30-39.

Tambasco, M., and Steinman, D. A. 2003. Path-dependent hemodynamics of the stenosed carotid bifurcation, Annals of Biomedical Engineering, 31: 1054-65.

Torii, R., Wood, N. B., Hadjiloizou, N., Dowsey, A. W., Wright, A. R., Hughes, A. D., Davies, J., Francis, D. P., Mayet, J., Yang, G.-Z., Thom, S. A. M., and Xu, X. Y. 2009. Fluidstructure interaction analysis of a patient-specific right coronary artery with physiological velocity and pressure waveforms, Int J Numer Method Biomed Eng, 25: 565-80.

Usmani, A. Y., and Muralidhar, K. 2016. Pulsatile flow in a compliant stenosed asymmetric model, Experiments in Fluids, 57: 186-210.

Valen-Sendstad, K., and Steinman, D. A. 2014. Mind the gap: Impact of computational fluid dynamics solution strategy on prediction of intracranial aneurysm hemodynamics and rupture status indicators, AJNR Am J Neuroradiol, 35: 536-43.

Varghese, S. S., Frankel, S. H., and Fischer, P. F. 2008. Modeling transition to turbulence in eccentric stenotic flows, Journal of Biomechanical Engineering, 130: 014503.

Waller, B. F. 1989. The eccentric coronary atherosclerotic plaque: Morphologic observations and clinical relevance, Clin Cardiol, 12: 14-20.

Womersley, J. R. 1955. Method for the calculation of velocity, rate of flow and viscous drag in arteries when the pressure gradient is known, The Journal of Physiology, 127: 55363.

Young, D. F., Cholvin, N. R., and Roth, A. C. 1975. Pressure drop across artificially induced stenoses in the femoral arteries of dogs, Circulation Research, 36: 735-43.

Young, D. F., and Tsai, F. Y. 1973. Flow characteristics in models of arterial stenoses - i. Steady flow, Journal of Biomechanics, 6: 395-410.

Zhang, J. M., Luo, T., Tan, S. Y., Lomarda, A. M., Wong, A. S., Keng, F. Y., Allen, J. C., Huo, Y., Su, B., Zhao, X., Wan, M., Kassab, G. S., Tan, R. S., and Zhong, L. 2015. Hemodynamic analysis of patient-specific coronary artery tree, Int $J$ Numer Method Biomed Eng, 31: e02708.

Zhu, C., Seo, J.-H., and Mittal, R. 2018. Computational modelling and analysis of haemodynamics in a simple model of aortic stenosis, Journal of Fluid Mechanics, 851: 23-49. 
Tables

Table 1: Different cases considered to study the laminar-turbulent blood flow transition of a pulsatile flow.

\begin{tabular}{|c|c|c|c|c|c|c|}
\hline No. & Time period & Flow rate $(\mathbf{m l} / \mathbf{s})$ & Velocity $\mathbf{( m / s )}$ & Degree of stenosis $\mathbf{( \% )}$ & $\mathbf{R e}$ & Wo \\
\hline $\mathbf{1}$ & 1 beat/s $(\mathrm{T}=1)$ & 1.1 & 0.155618 & 67 & 146 & 2.10 \\
\hline $\mathbf{2}$ & 2 beat/s $(\mathrm{T}=0.5)$ & 2.5 & 0.353678 & 67 & 331 & 2.97 \\
\hline $\mathbf{3}$ & 3 beat/s $(\mathrm{T}=0.33)$ & 3.9 & 0.551737 & 67 & 516 & 3.64 \\
\hline $\mathbf{4}$ & 3 beat/s $(\mathrm{T}=0.33)$ & 3.9 & 0.551737 & 16 & 516 & 3.64 \\
\hline $\mathbf{5}$ & 3 beat $/ \mathrm{s}(\mathrm{T}=0.33)$ & 3.9 & 0.551737 & 39.1 & 516 & 3.64 \\
\hline
\end{tabular}

Table 2: The maximum values of area-weighted average of TKE for different cases considered in this study.

\begin{tabular}{|c|c|c|c|c|c|c|c|}
\hline & \multirow{2}{*}{$\begin{array}{c}\text { Flow } \\
\text { condition }\end{array}$} & \multirow{2}{*}{$\begin{array}{l}\text { Flow } \\
\text { rate } \\
(\mathrm{ml} / \mathrm{s})\end{array}$} & \multirow{2}{*}{$\begin{array}{c}\text { Degree of } \\
\text { stenosis } \\
(\%)\end{array}$} & \multicolumn{4}{|c|}{ Area-weighted average of TKE $(\mathrm{m} / \mathrm{s})^{2}$} \\
\hline & & & & $\begin{array}{l}\text { Maximum } \\
\text { deceleration }\end{array}$ & $\begin{array}{l}\text { Minimum } \\
\text { velocity }\end{array}$ & $\begin{array}{l}\text { Maximum } \\
\text { acceleration }\end{array}$ & $\begin{array}{c}\text { Maximum } \\
\text { velocity }\end{array}$ \\
\hline Case 1 & Resting & 1.1 & $\begin{array}{l}\text { Severe (67 } \\
\%)\end{array}$ & 0.006 & 0.004 & 0.01 & 0.05 \\
\hline Case 2 & In between & 2.5 & $\begin{array}{c}\text { Severe (67 } \\
\%)\end{array}$ & 0.03 & 0.02 & 0.05 & 0.23 \\
\hline Case 3 & Hyperemic & 3.9 & $\begin{array}{c}\text { Severe (67 } \\
\%)\end{array}$ & 0.07 & 0.05 & 0.13 & 0.59 \\
\hline Case 4 & Hyperemic & 3.9 & $\begin{array}{c}\text { Early stage } \\
(16 \%)\end{array}$ & 0.006 & 0.004 & 0.01 & 0.05 \\
\hline Case 5 & Hyperemic & 3.9 & $\begin{array}{c}\text { Moderate } \\
(39.1 \%) \\
\end{array}$ & 0.04 & 0.03 & 0.06 & 0.25 \\
\hline
\end{tabular}




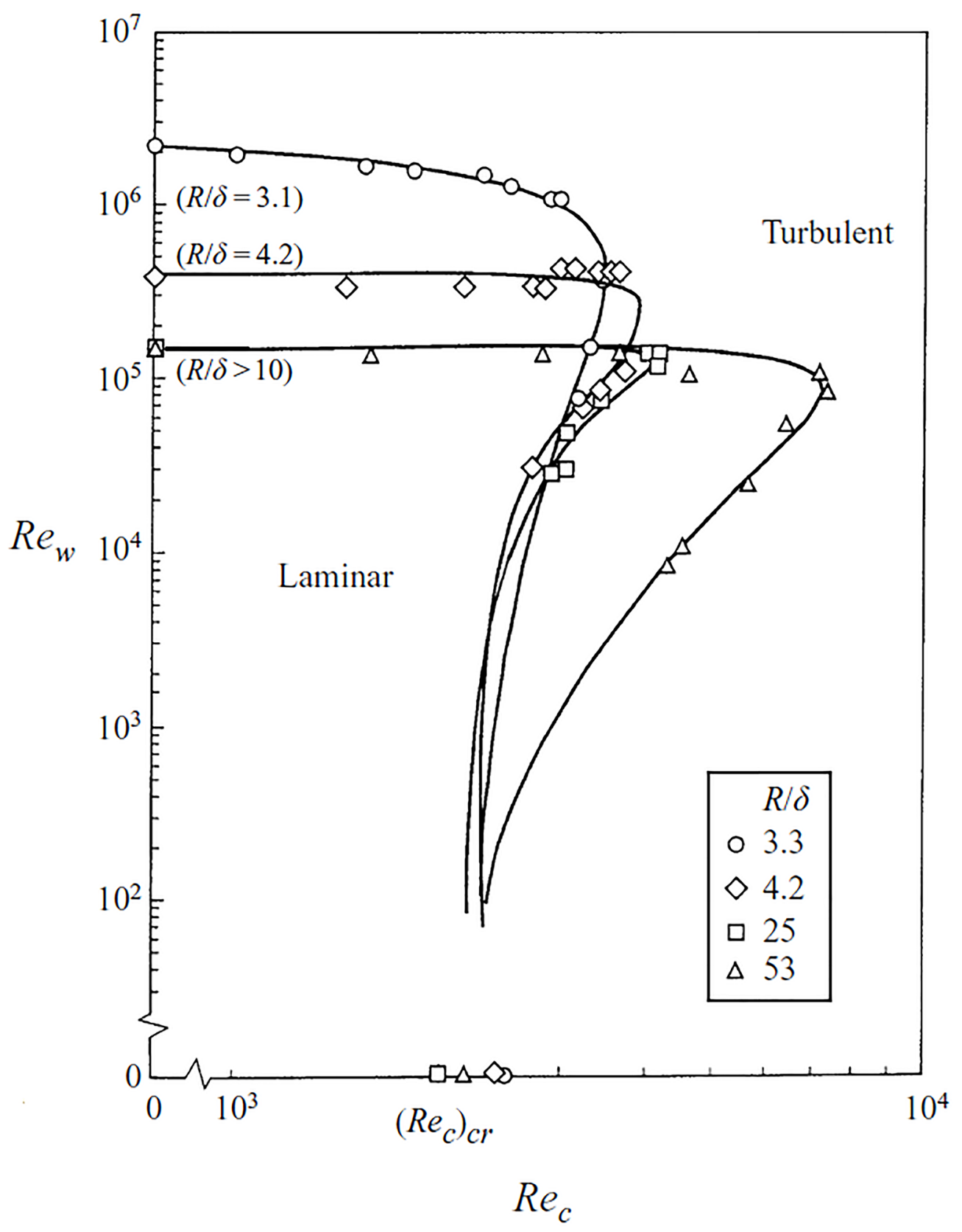

CNM_3347_fig1.tif 


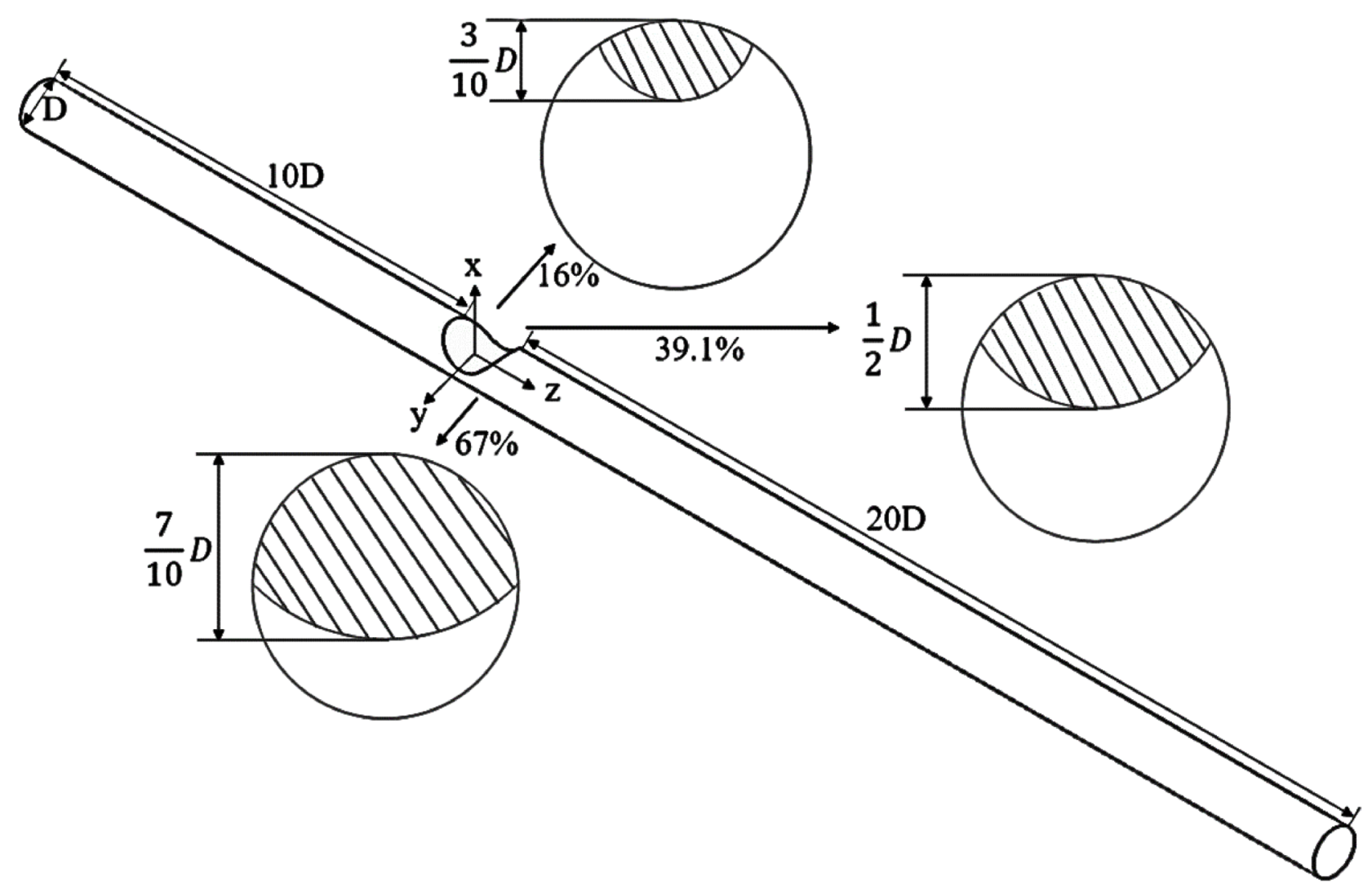

CNM_3347_fig2.tif 


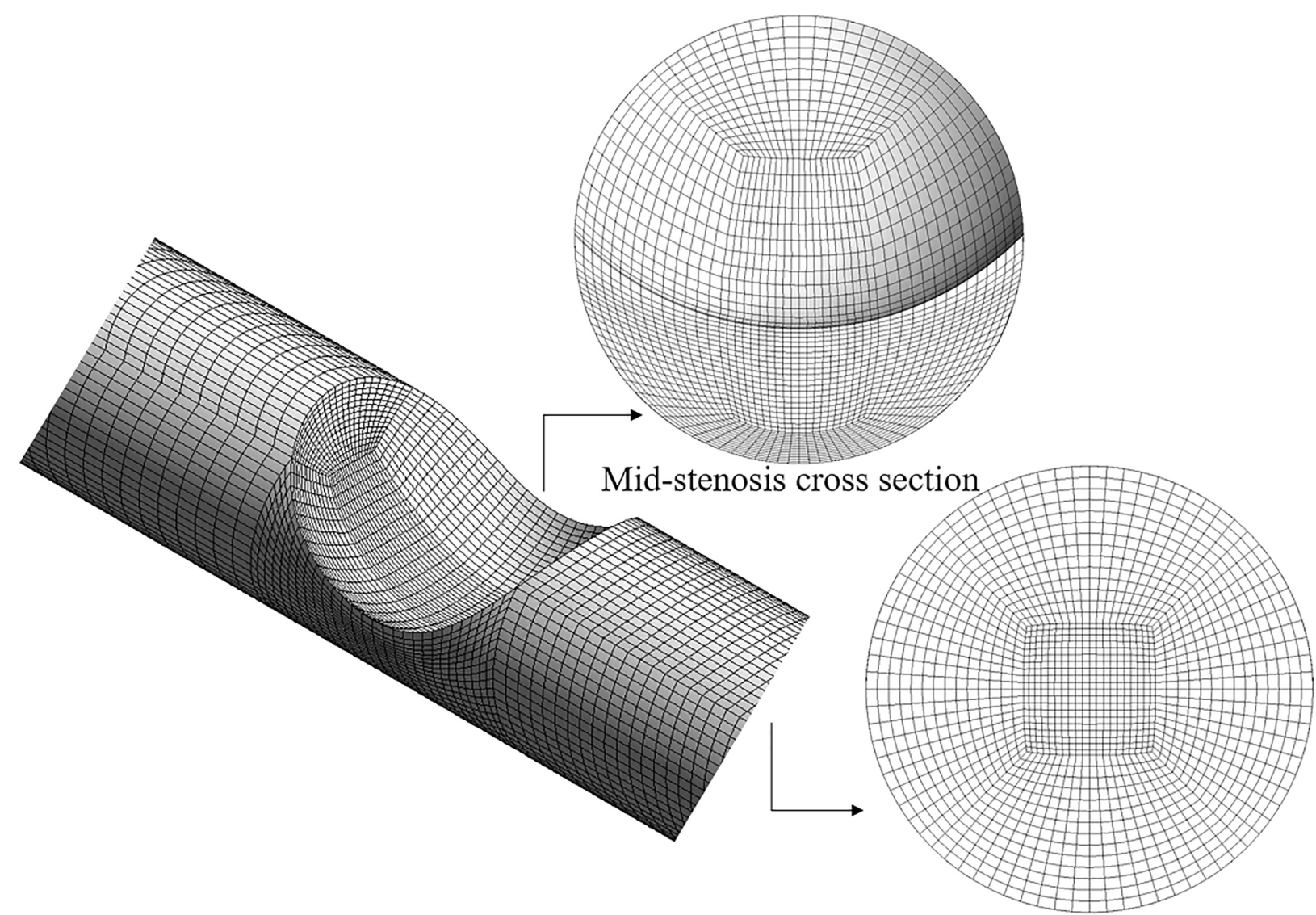

Coronary artery model cross section CNM_3347_fig3.tif

This article is protected by copyright. All rights reserved. 


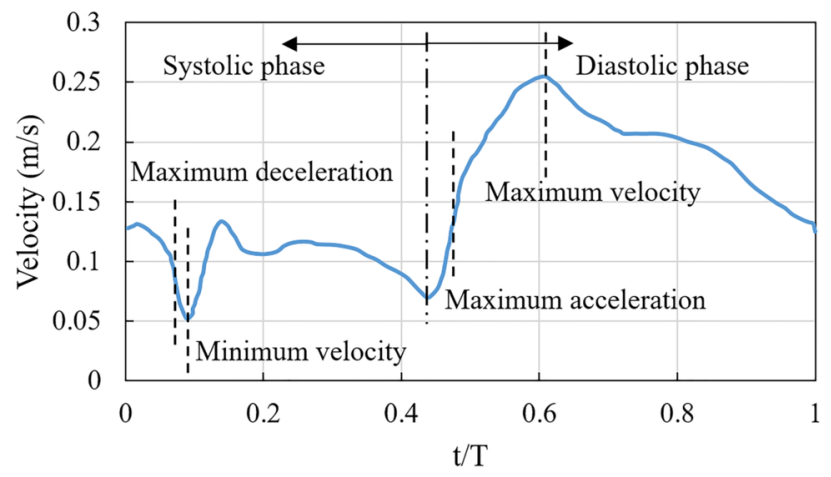

CNM_3347_fig4.tif

This article is protected by copyright. All rights reserved. 


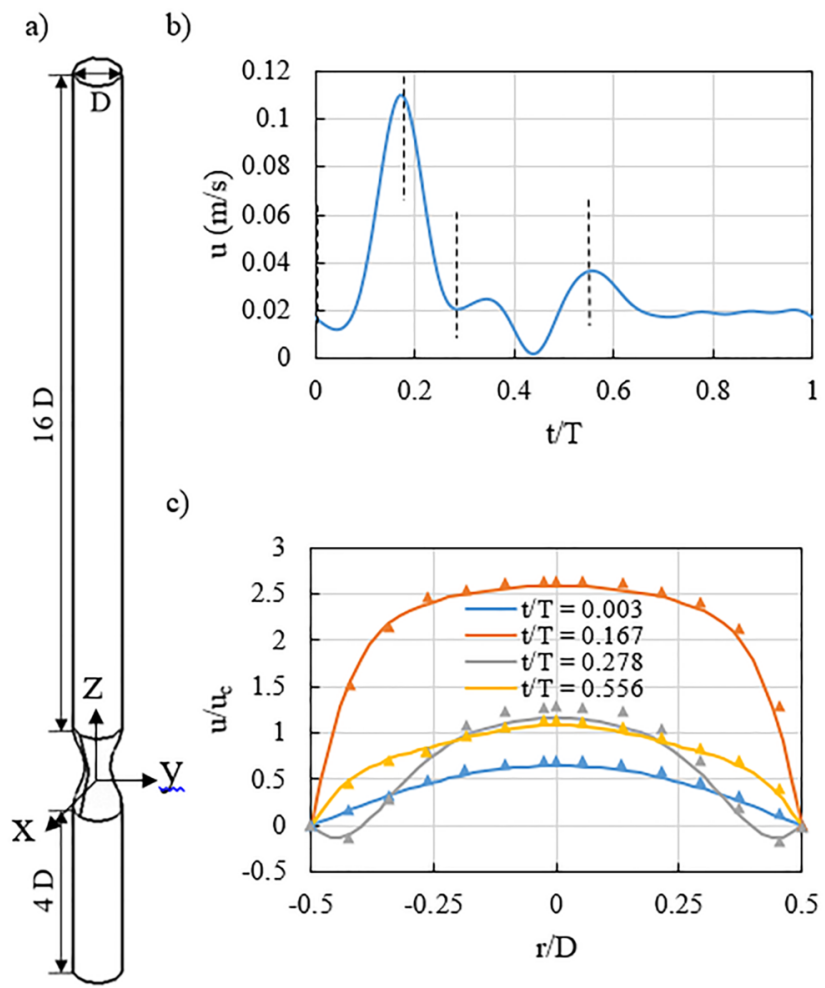

d)

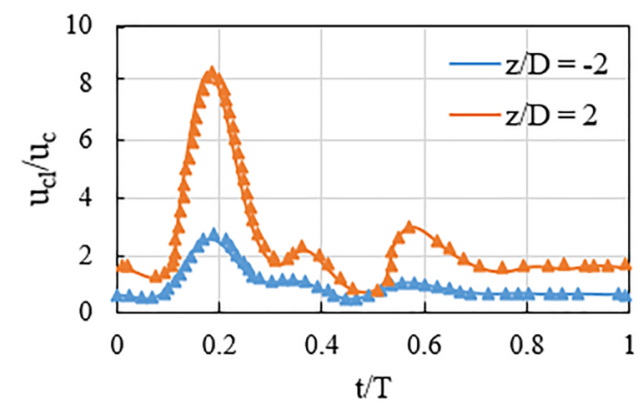

CNM_3347_fig5.tif

This article is protected by copyright. All rights reserved. 


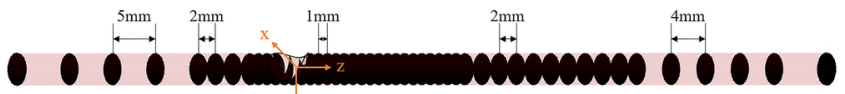

CNM_3347_fig6.tif

This article is protected by copyright. All rights reserved. 


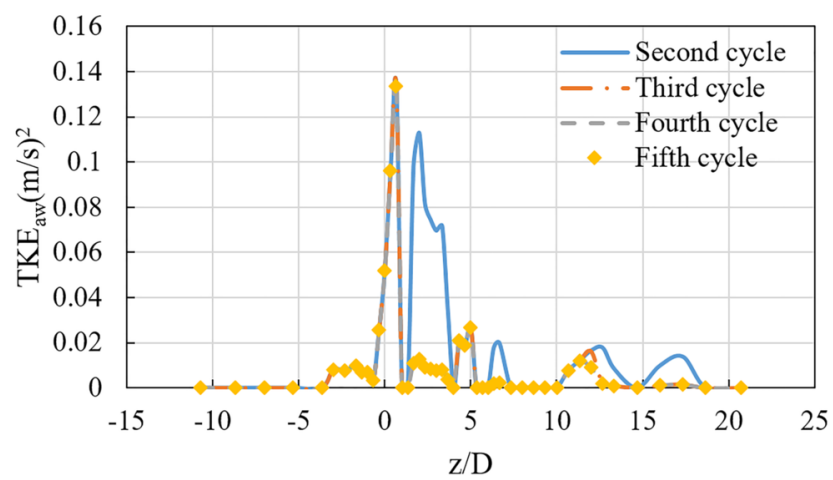

CNM_3347_fig7.tif

This article is protected by copyright. All rights reserved. 


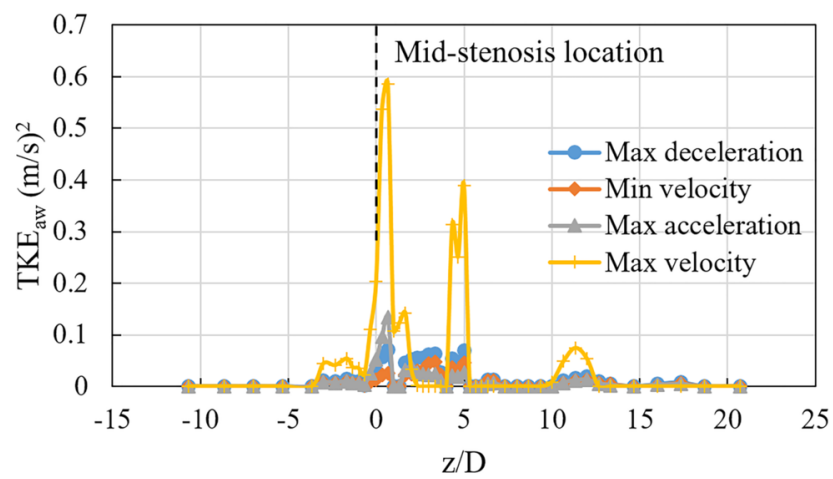

CNM_3347_fig8.tif

This article is protected by copyright. All rights reserved. 


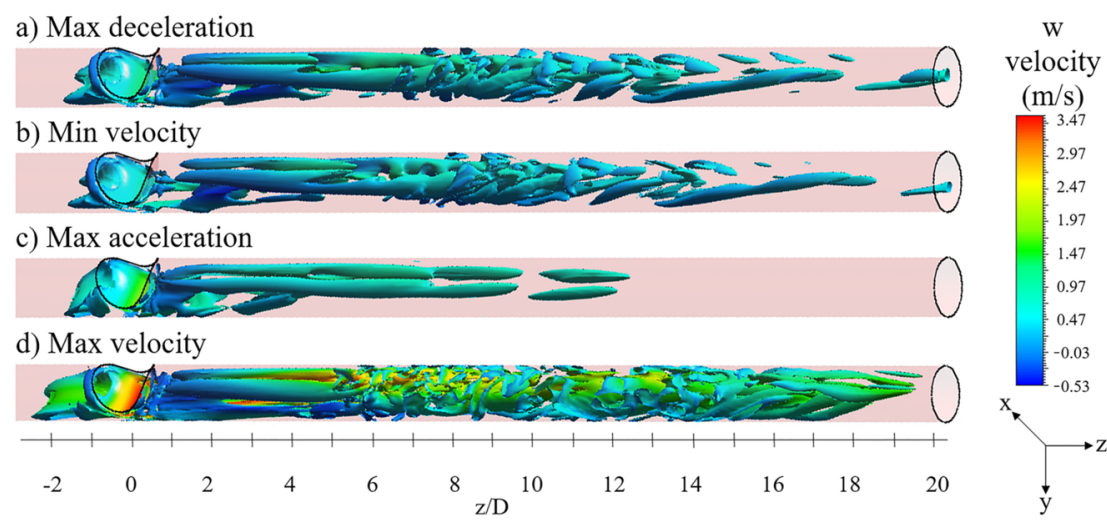

CNM_3347_fig9.tif

This article is protected by copyright. All rights reserved. 

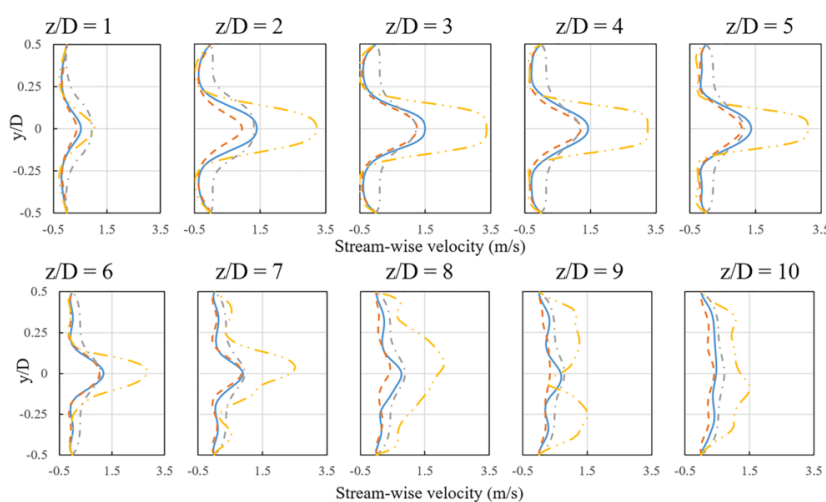

— Max deceleration - - Min velocity - - - Max acceleration _- Max velocity

CNM_3347_fig10.tif

This article is protected by copyright. All rights reserved. 


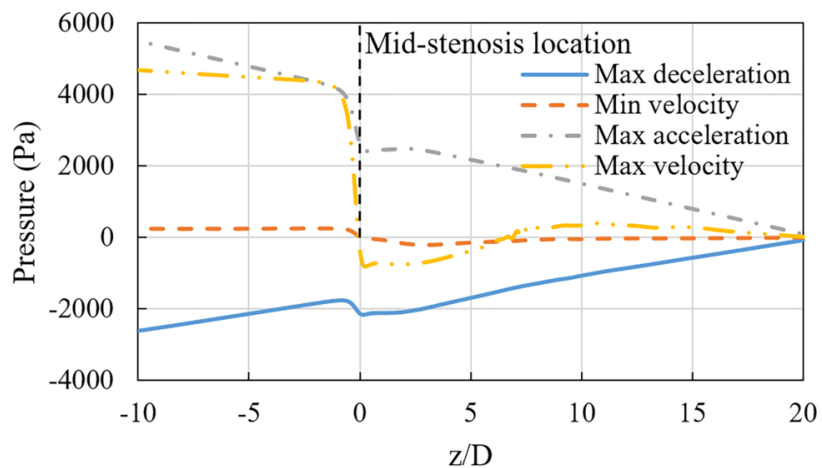

CNM_3347_fig11.tif

This article is protected by copyright. All rights reserved. 


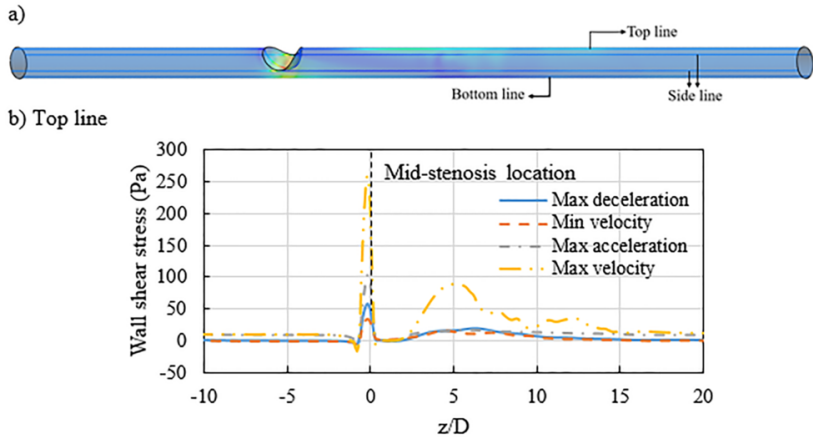

c) Side line



d) Bottom line

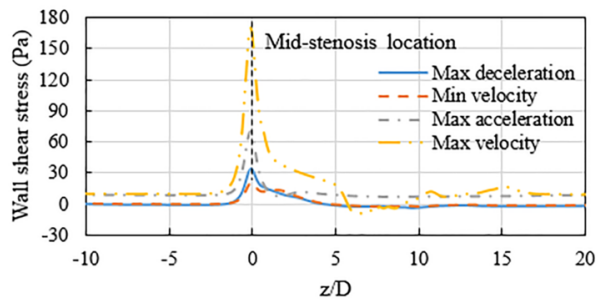

CNM_3347_fig12.tif

This article is protected by copyright. All rights reserved. 


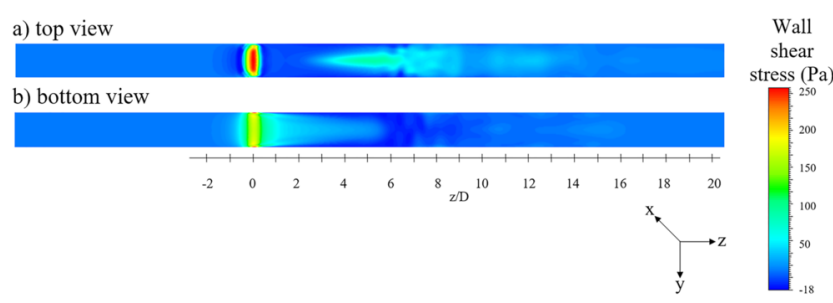

CNM_3347_fig13.tif

This article is protected by copyright. All rights reserved. 

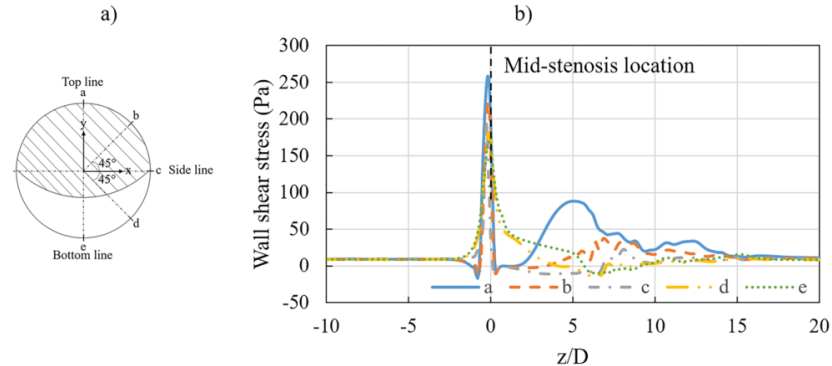

CNM_3347_fig14.tif

This article is protected by copyright. All rights reserved. 


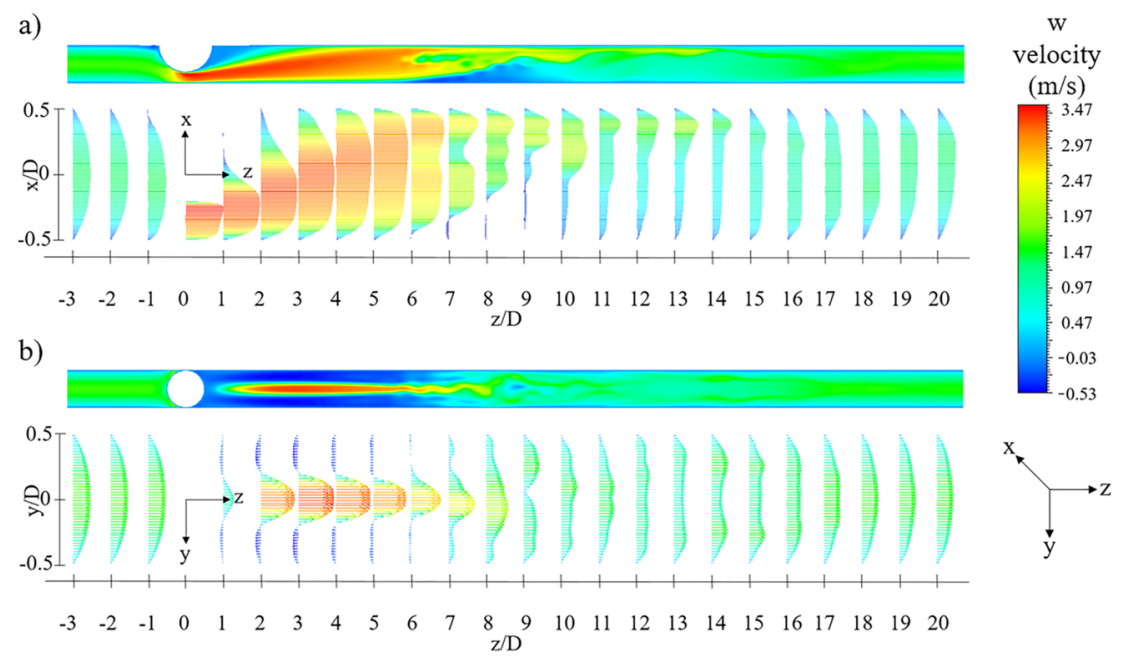

c)

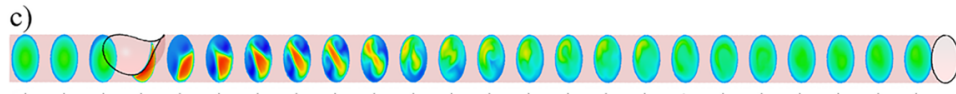

$\begin{array}{lllllllllllllllllllllllll}-3 & -2 & -1 & 0 & 1 & 2 & 3 & 4 & 5 & 6 & 7 & 8 & 9 & 10 & 11 & 12 & 13 & 14 & 15 & 16 & 17 & 18 & 19 & 20\end{array}$

CNM_3347_fig15.tif

This article is protected by copyright. All rights reserved. 
a)



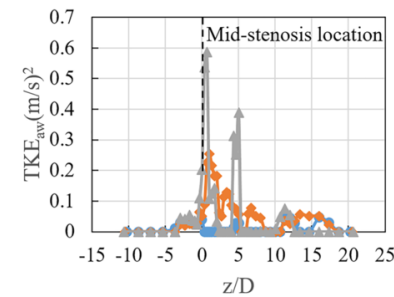

$\multimap-16 \% \rightarrow 39.1 \% \rightarrow 67 \%$

CNM_3347_fig16.tif

This article is protected by copyright. All rights reserved. 


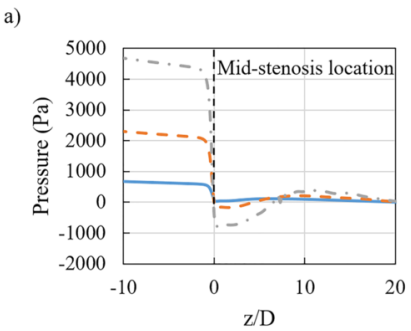

b)

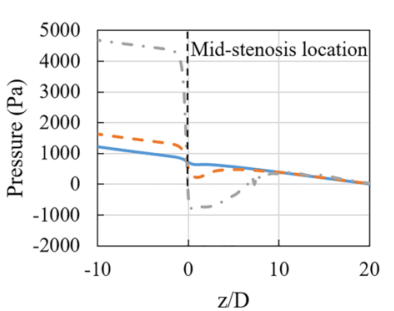

—Case 1 - - - Case 2 - - Case 3

$-16 \%---39.1 \%-\cdot-67 \%$

CNM_3347_fig17.tif

This article is protected by copyright. All rights reserved. 

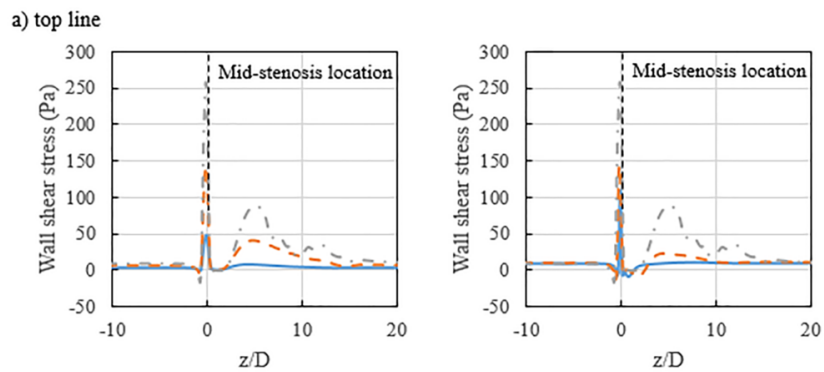

b) side line
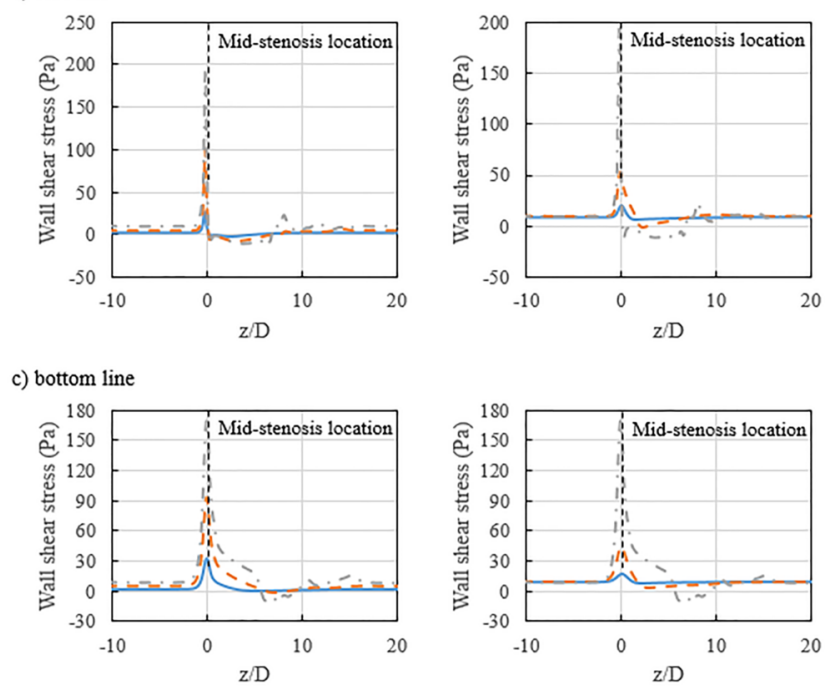

_Case 1 - - - Case 2 - - - Case 3

$-16 \%---39.1 \%-\cdot-67 \%$

CNM_3347_fig18.tif

This article is protected by copyright. All rights reserved. 


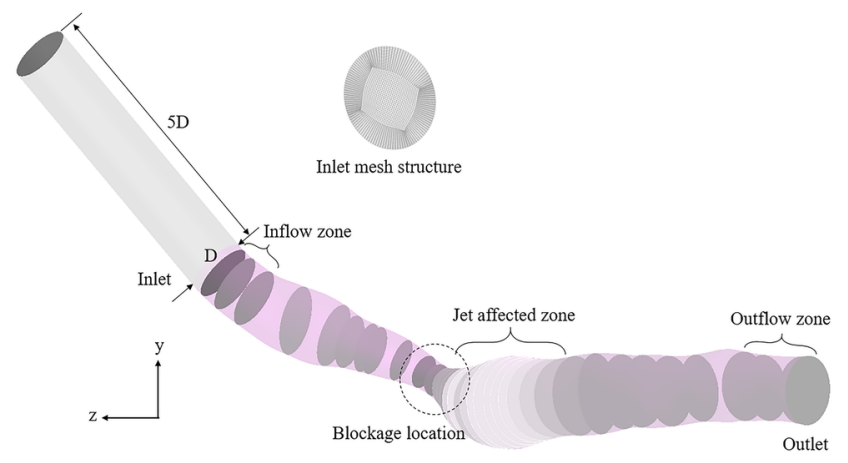

CNM_3347_fig19.tif

This article is protected by copyright. All rights reserved. 


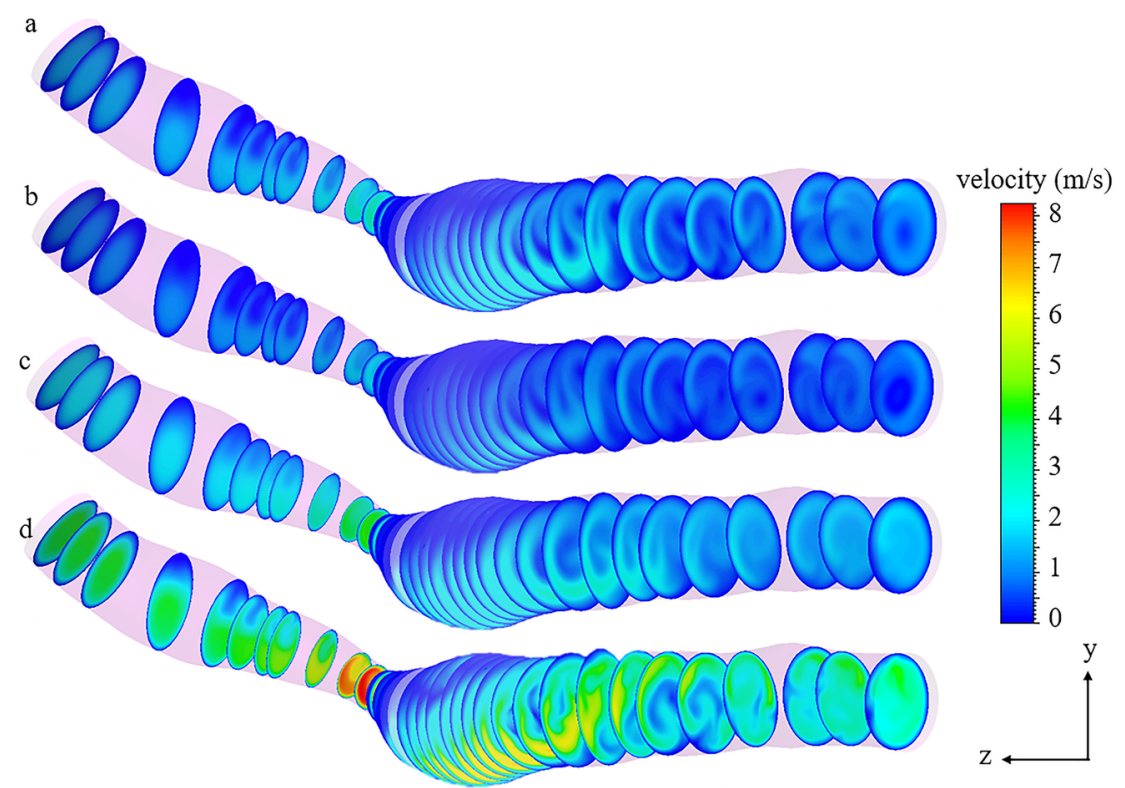

CNM_3347_fig20.tif

This article is protected by copyright. All rights reserved. 

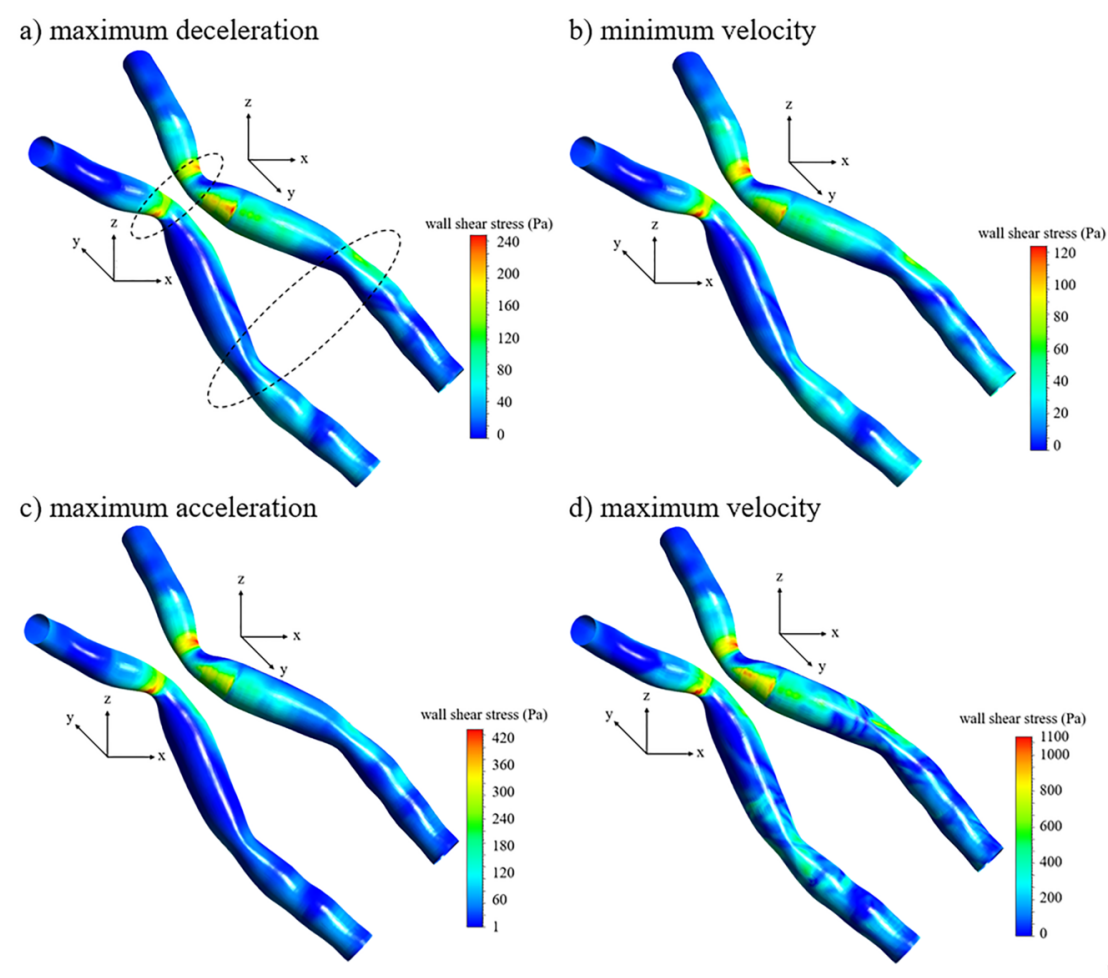

d) maximum velocity

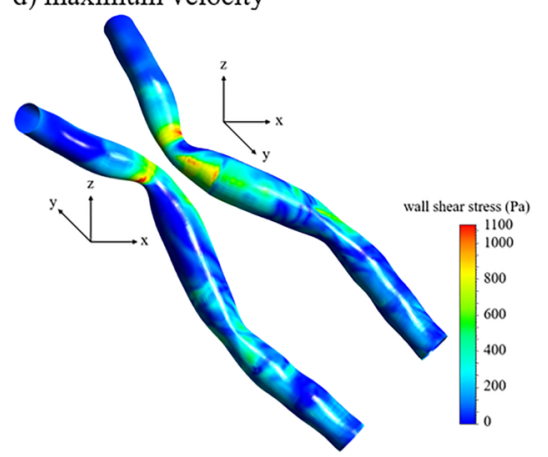

CNM_3347_fig21.tif

This article is protected by copyright. All rights reserved. 


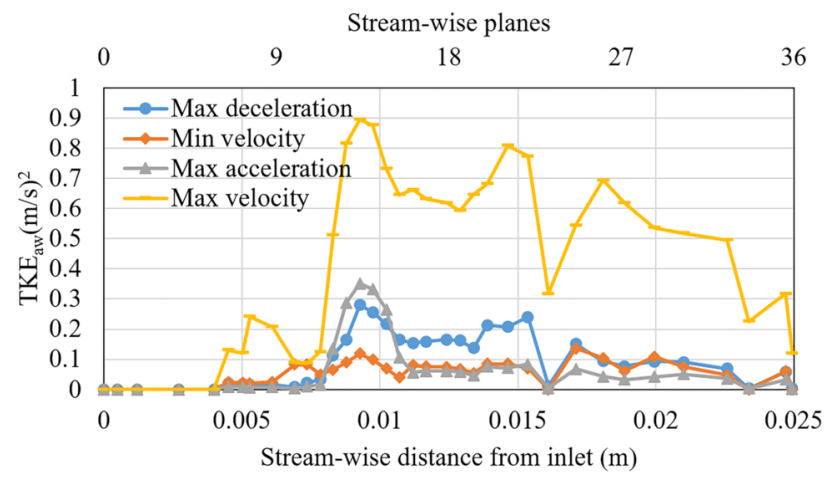

CNM_3347_fig22.tif

This article is protected by copyright. All rights reserved. 


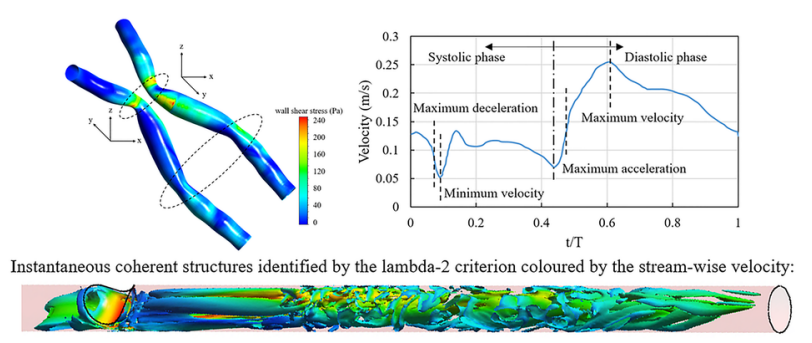

CNM_3347_graphical abstract.tif

This article is protected by copyright. All rights reserved. 
- Asymmetric stenosis inside a coronary artery model has been investigated.

- Turbulence inside a diseased coronary artery model with a physiological pulsatile flow has been studied.

- A susceptible location for second stenosis has been reported.

- A second stenosis is likely to be formed 5D to 10D downstream of the first stenosis. 


\section{University Library}

\section{- M M I N E R VA A gateway to Melbourne's research publications}

Minerva Access is the Institutional Repository of The University of Melbourne

\section{Author/s:}

Freidoonimehr, N;Arjomandi, M;Sedaghatizadeh, N;Chin, R;Zander, A

Title:

Transitional turbulent flow in a stenosed coronary artery with a physiological pulsatile flow

Date:

2020-07

Citation:

Freidoonimehr, N., Arjomandi, M., Sedaghatizadeh, N., Chin, R. \& Zander, A. (2020).

Transitional turbulent flow in a stenosed coronary artery with a physiological pulsatile flow. International Journal for Numerical Methods in Biomedical Engineering, 36 (7), https:// doi.org/10.1002/cnm.3347.

Persistent Link:

http://hdl.handle.net/11343/275789 